Review

\title{
Synthesis of the Marine Pyrroloiminoquinone Alkaloids, Discorhabdins
}

\section{Yasufumi Wada ${ }^{1, \dagger}$, Hiromichi Fujioka ${ }^{1, *}$ and Yasuyuki Kita ${ }^{1,2, *}$}

1 Graduate School of Pharmaceutical Sciences, Osaka University, 1-6 Yamada-oka, Suita, Osaka, 565-0871, Japan

2 College of Pharmaceutical Sciences, Ritsumeikan University, 1-1-1 Nojihigashi, Kusatsu, Shiga, 525-8577, Japan

$\dagger$ JSPS Research Fellow.

* Authors to whom correspondence should be addressed; E-Mails: fujioka@ phs.osaka-u.ac.jp (H.F.); kita@ph.ritsumei.ac.jp (Y.K.); Tel.: +81 66879 8225; Fax: +81 668798229.

Received: 7 April 2010; in revised form: 16 April 2010 / Accepted: 19 April 2010 /

Published: 21 April 2010

\begin{abstract}
Many natural products with biologically interesting structures have been isolated from marine animals and plants such as sponges, corals, worms, etc. Some of them are discorhabdin alkaloids. The discorhabdin alkaloids (discorhabdin A-X), isolated from marine sponges, have a unique structure with azacarbocyclic spirocyclohexanone and pyrroloiminoquinone units. Due to their prominent potent antitumor activity, discorhabdins have attracted considerable attention. Many studies have been reported toward the synthesis of discorhabdins. We have accomplished the first total synthesis of discorhabdin A (1), having the strongest activity in vitro among discorhabdins in 2003. In 2009, we have also accomplished the first total synthesis of prianosin B (2), having the 16,17dehydropyrroloiminoquinone moiety, by a novel dehydrogenation reaction with a catalytic amount of $\mathrm{NaN}_{3}$. These synthetic studies, as well as syntheses of the discorhabdins by various chemists to-date, are reviewed here.
\end{abstract}

Keywords: discorhabdin alkaloid; marine sponge; cytotoxic activity 


\section{Introduction}

Marine organisms contain various elements that are important for their survival and for their relationships with other living bodies. The nature, action and role of many of these elements have not yet been elucidated. Many marine organic compounds have been shown to be also useful for humans; as drugs, agricultural medicines, cosmetics and health foods [1-5]. In particular, a number of marine drugs have been developed in recent years. Marine natural products offer challenging targets to synthetic chemists due to their complicated structures that exhibit remarkable biological activities [6-11]. However, the chemical synthesis of marine drugs is of upmost importance as a synthetic supply is needed when it is difficult to maintain the amount of the natural source.

Figure 1. The structure of discorhabdin alkaloids.
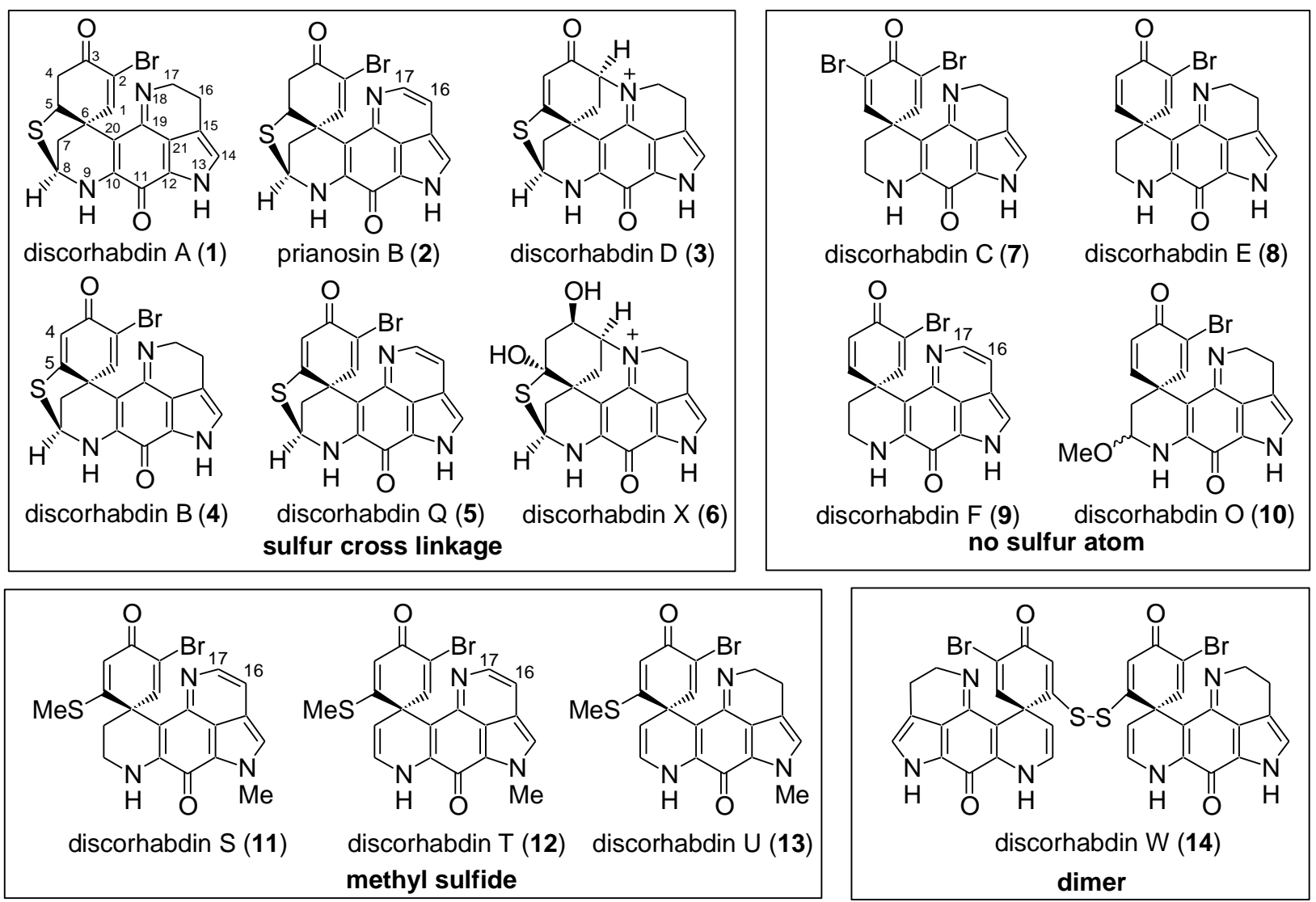

In the past several years, many new pyrroloiminoquinone alkaloids have been isolated from marine sources. The pyrroloiminoquinone alkaloid family consists of discorhabdins [12-16], prianosins [17], makaluvamines [18-21], batzellins [22-23], wakayins [24] and damirones [25-26], and have potent antitumor activities derived from their unique fused ring structures [27]. Among them, the discorhabdin alkaloids were isolated from marine sponges such as New Zealand sponges of the genus Latrunculia, the Okinawan sponge Prianos melanos, the Fijian sponge Zyzzya cf. Marsailis, etc. [12-16]. All of them have unique structures, with azacarbocyclic spirocyclohexanone and pyrroloiminoquinone structures. Figure 1 shows some representative discorhabdin alkaloids. Among the various isolated discorhabdins (A-X), discorhabdins A (1) [28,29], B (4) [28,29], D (3) [30], H [15], I [14], J [12], L [14], M [12], N [12], Q (5) [31], R [32], X (6) [33], and prianosins B (2), D [34] 
have a sulfur-containing fused ring structure. Discorhabdins S (11), T (12) and U (13) [13] have a methyl sulfide moiety. Discorhabdin W (14) [35] is a dimeric structure with a disulfide bond, while the others have no sulfur atom. Furthermore, discorhabdins F (9) [36], Q, S, T and prianosin B (2) contain a 16,17-dehydropyrroloiminoquinone moiety. Because of their prominent potent antitumor activity and unusual ring structure, pyrroloiminoquinone alkaloid synthesis has attracted the interest of many organic chemists, and over the last decade, the total synthesis of a few of them as well as synthetic approaches has appeared. Our group accomplished the first total syntheses of discorhabdin C (7) [37], makaluvamine F [38,39] and discorhabdin A (1) [40-43]. In 2009, we also accomplished the first total synthesis of prianosin B (2) [44]. In this review, we describe the total syntheses of the discorhabdins by various chemists to-date.

\section{Studies of the Total Synthesis of Discorhabdin C}

In 1986, discorhabdin C (7) was isolated from the sponge Latrunculia in New Zealand by Munro et al. and was found to exhibit a strong cytotoxicity against tumor cells $\left(\mathrm{ED}_{50}=30 \mathrm{ng} / \mathrm{mL}\right.$ against P388 and <100 ng/mL against L1210) [45]. Discorhabdin C was the first isolated of the discorhabdins and much attention has been paid to the total synthesis of this attractive target.

\subsection{Our Synthesis [37]}

The problems in the synthesis of discorhabdin $\mathrm{C}$ are the construction of highly fused ring systems and the formation of the indoloquinone imine moiety. An overview of our approaches to the synthesis of discorhabdin $\mathrm{C}$ is illustrated in Scheme 1. One approach involves the imine formation between the tryptamine amine of the side chain and the indoloquinone carbonyl as the final step of the synthesis (route a). Another approach involves the oxidative coupling of the indoloquinone imine by phenyliodine bistrifluoroacetate (PIFA) as the final step (route b) [46].

Scheme 1. Retrosynthetic analysis for discorhabdin C.

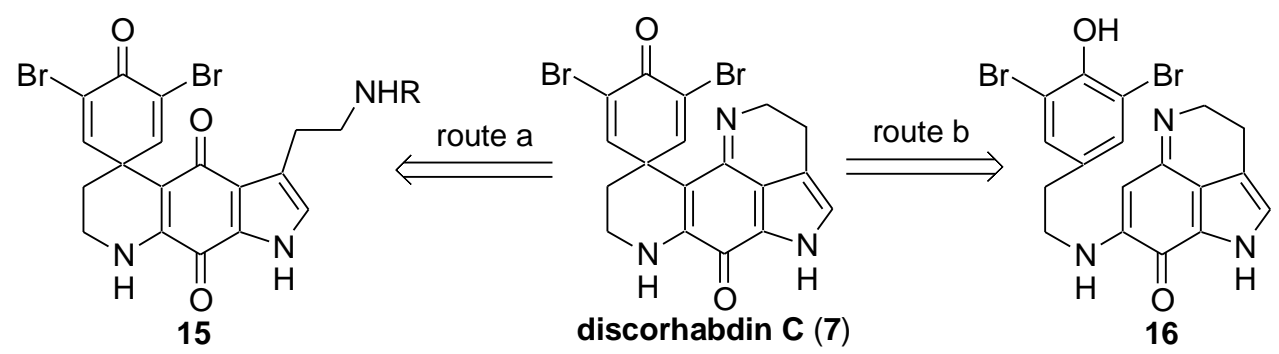

A reasonable starting material for route a is 2-hydroxy-4-methoxybenzaldehyde (17). The benzylation of 17 with $\mathrm{BnBr}$ and $\mathrm{K}_{2} \mathrm{CO}_{3}$ followed by condensation with ethyl azidoacetate $\left(\mathrm{N}_{3} \mathrm{CH}_{2} \mathrm{CO}_{2} \mathrm{Et}\right)$ in ethanolic sodium ethoxide gave the vinyl azide, which was decomposed in boiling xylene to give the 2-(ethoxycarbonyl)indole (18) in $73 \%$ yield over three steps. Hydrolysis of the ester group of 18 with $\mathrm{KOH}$ gave indolecarboxylic acid, which was decarboxylated with copper chromite under thermal conditions to give the 4,6-disubstituted indole (19). The treatment of the indole 19 with dimethyl(methylene)ammonium iodide $\left(\mathrm{CH}_{2}=\mathrm{N}^{+} \mathrm{Me}_{2} \mathrm{I}^{-}\right)$gave the 3-(dimethylamino)methyl derivative. The dimethylamino group was replaced by a cyano group using $\mathrm{NaCN}$ via the quaternary salt to yield 
the 3-(cyanomethyl)indole (20). Catalytic hydrogenation of the cyano group of $\mathbf{2 0}$ with Raney $\mathrm{Ni}$ followed by protection of the resulting amino group with the trifluoroacetyl or the [(trimethylsilyl)ethoxy]carbonyl (Teoc) groups afforded 21a $\left(\mathrm{R}=\mathrm{COCF}_{3}\right)$ and $\mathbf{2 1 b}(\mathrm{R}=\mathrm{Teoc})$, respectively. Debenzylation of 21a,b with reduction followed by oxidation using Fremy's salt gave the corresponding quinones $(\mathbf{2 2 a}, \mathbf{b})$. The treatment of $\mathbf{2 2 a}, \mathbf{b}$ with 3,5-debromotyramine hydrobromide gave the phenol derivatives (23a,b). Silylation of 23a,b with $o$-silylated ketene acetal $\left(\mathrm{MeCH}=\mathrm{C}(\mathrm{OMe})\left(\mathrm{OSiMe}_{3}\right)\right)$ followed by oxidation with PIFA resulted in the desired intermediate $(\mathbf{1 5 a}, \mathbf{b})$. Unfortunately, all attempts to effect the final imine formation between the tryptamine nitrogen and the indoloquinone carbonyl in these types of intermediates failed (Scheme 2).

Scheme 2. The synthetic approach toward discorhabdin $\mathrm{C}$ via route a.

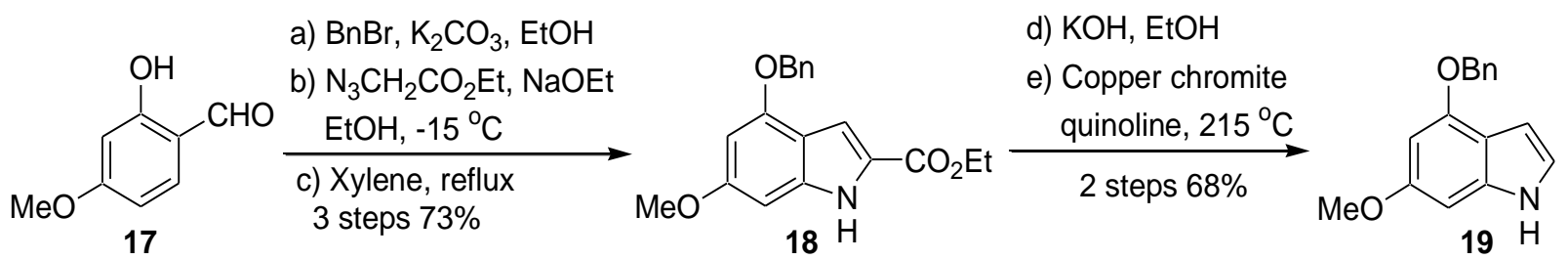

$$
\begin{aligned}
& \text { f) } \mathrm{CH}_{2}=\mathrm{N}^{+} \mathrm{Me}_{2} \mathrm{I}^{-}, \mathrm{CH}_{2} \mathrm{Cl}_{2} \\
& \text { g) } \mathrm{Mel}, 0{ }^{\circ} \mathrm{C}
\end{aligned}
$$

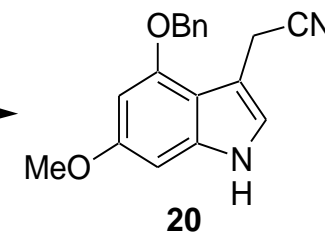

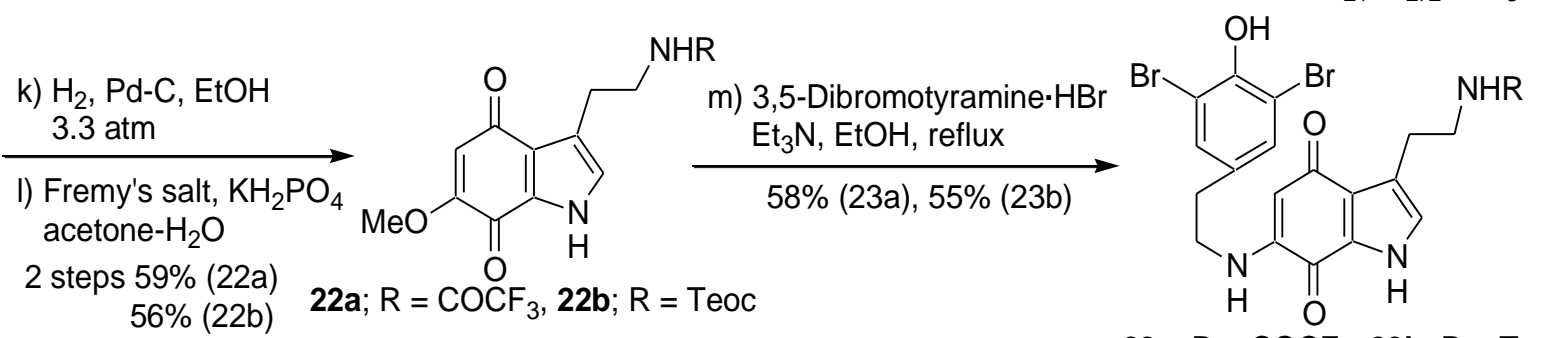
$56 \%(22 b)$

22a; $R=\mathrm{COCF}_{3}, \mathbf{2 2 b} ; \mathrm{R}=\mathrm{TeOC}$

23a; $R=\mathrm{COCF}_{3}, \mathbf{2 3 b} ; \mathrm{R}=\mathrm{TeOC}$

n) $\mathrm{MeCH}=\mathrm{C}(\mathrm{OMe})\left(\mathrm{OSiMe}_{3}\right)$ $\mathrm{CH}_{2} \mathrm{Cl}_{2}$, rt

o) PIFA, $\mathrm{CF}_{3} \mathrm{CH}_{2} \mathrm{OH}$, rt

2 steps $58 \%(15 a)$

$62 \%(15 b)$

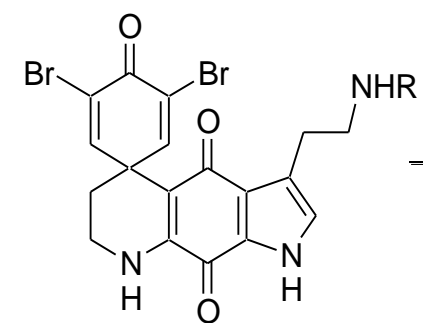

$\stackrel{\text { NHR }}{\longrightarrow}$ discorhabdin C (7)

$15 a ; R=\mathrm{COCF}_{3}, 15 b ; \mathrm{R}=\mathrm{TeOc}$

An alternative approach (route b), in which phenolic coupling of the previously produced aminoindoloquinone imine (16) is employed in the final step, accomplished the first total synthesis of discorhabdin C (7). The direct imine formation from the indoloquinone was achieved by protection of the indoloquinone nitrogen with the tosyl group followed by an acidic dehydrative treatment. The treatment of 22b with $p$-toluenesulfonyl chloride gave the $N$-tosylate (24). Deprotection of the Teoc group of $\mathbf{2 4}$ with anhydrous $p$-toluenesulfonic acid in acetonitrile in the presence of $3 \AA$ molecular sieves and sodium bicarbonate yielded an unstable indoloquinone imine (25), which was subjected to 
the following one-pot transformation without further purification. When treated with 3,5-dibromotyramine hydrobromide in ethanol, the indoloquinone imine underwent a facile substitution reaction and subsequent detosylation to give the phenolic aminoindoloquinone imine (16). The conversion of $\mathbf{1 6}$ into its corresponding silylether and the subsequent oxidative coupling reaction using PIFA gave rise to discorhabdin C (7) (Scheme 3).

This efficient route was used for the synthesis of a wide variety of previously inaccessible sulfurcontaining discorhabdin and prianosin alkaloids (vide infra).

Scheme 3. The total synthesis of discorhabdin C via route b.
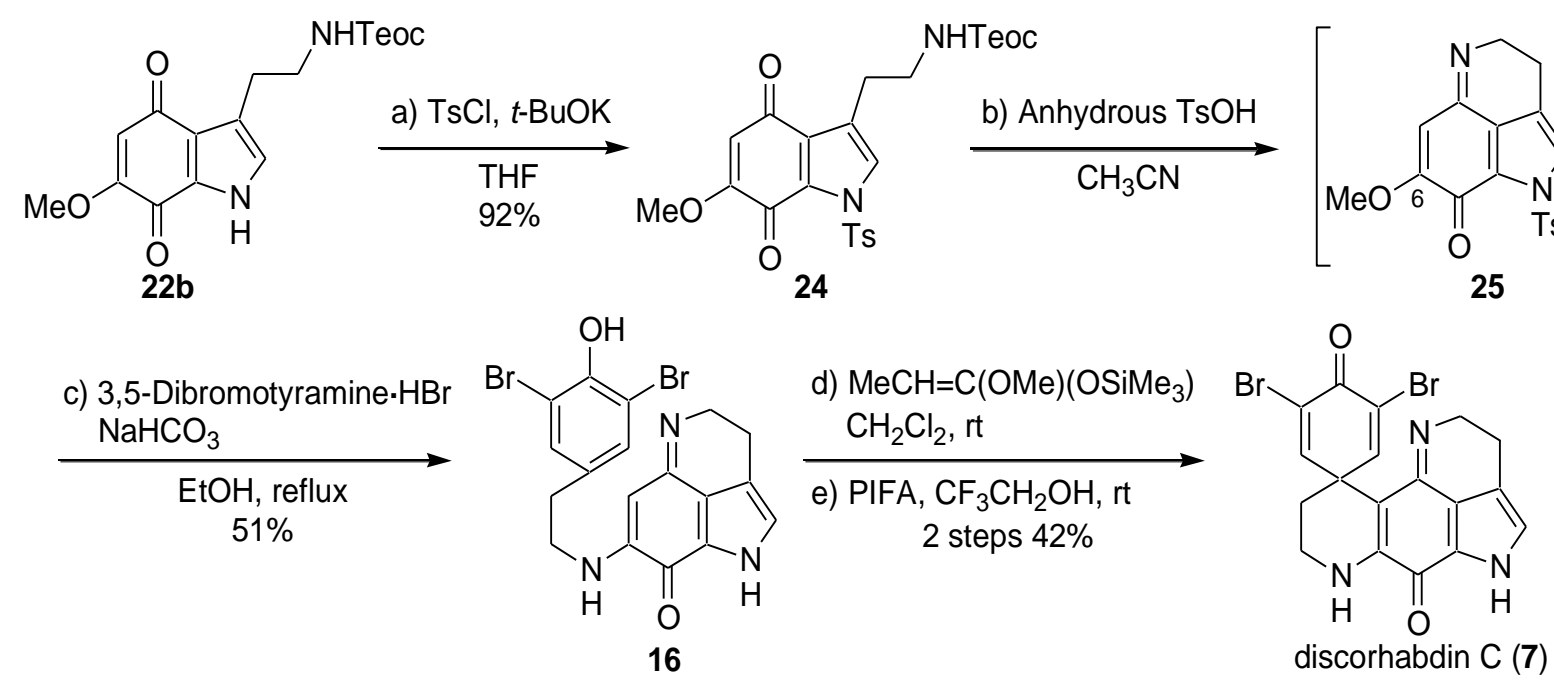

\subsection{Yamamura's Synthesis $[47,48]$}

At the same time as our report, Yamamura et al. also reported the total synthesis of discorhabdin C (7). Their synthesis was initiated from the conversion of the known nitrobenzaldehyde (26) into the corresponding amide compound (27) in a two-step procedure: reduction of the nitro group followed by $\mathrm{Z}$ protection of the generated amine. Manipulation of $\mathbf{2 7}$ involving the Curtius reaction provided the diaminobenzene, whose two amino functions could be differentiated by the diverse protection groups (Teoc and Z). Thus, after reductive removal of the benzyloxycarbonyl group, the generated amine was benzylated in the usual manner to give 29. Among a variety of methods for indole-skeleton constructions, they chose the reaction of $\mathbf{2 9}$ with ethyl 4-chloroacetoacetate, which gave rise to the simultaneous introduction of a $\mathrm{C}_{2}$ unit at the $\mathrm{C}-3$ position of the indole (30). Access to the tetrahydropyrroloquinoline (32) was effected by Gannortt's procedure, i.e., the stepwise removal of the amino and carboxylic acid protective groups of $\mathbf{3 0}$ followed by lactam formation provided the tricyclic product (31) in a good overall yield. The amide function of $\mathbf{3 1}$ was reduced with $\mathrm{BH}_{3} \cdot \mathrm{SMe}_{2}$, and the resulting amine was oxidized with CAN (ceric ammonium nitrate) to produce the expected tetrahydropyrroloquinoline (32) in two steps, which further underwent a nucleophilic substitution reaction with 3,5-dibromotyramine to furnish 33 in 78\% yield. The electrolysis of bromophenol (33) furnished the expected benzyldiscorhabdin $\mathrm{C}(\mathbf{3 4})$ accompanied by the ring-expanded product (35) (Scheme 4). 
Scheme 4. The synthesis of benzyldiscorhabdin C by Yamamura et al.

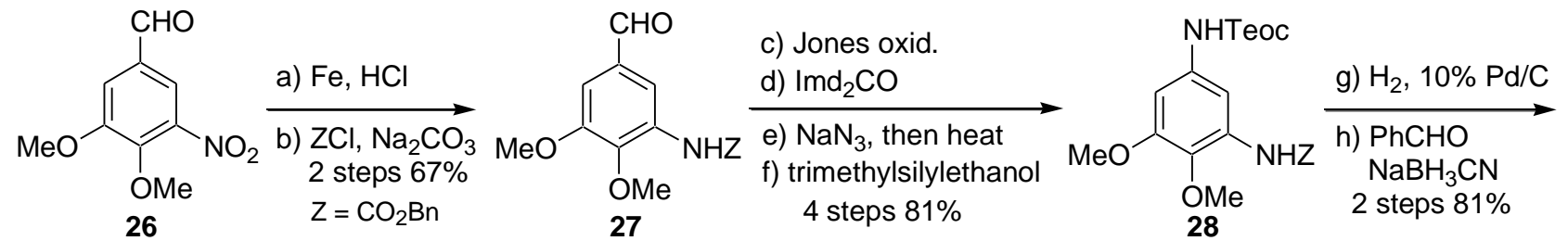

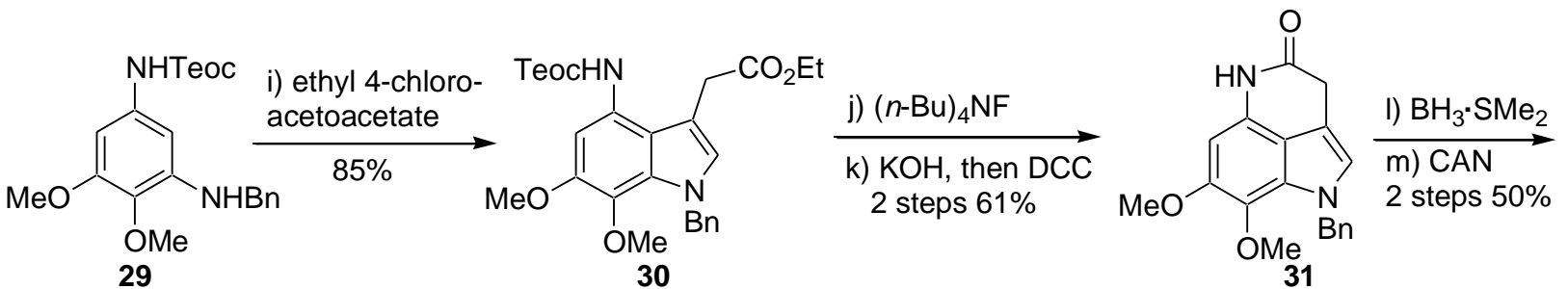

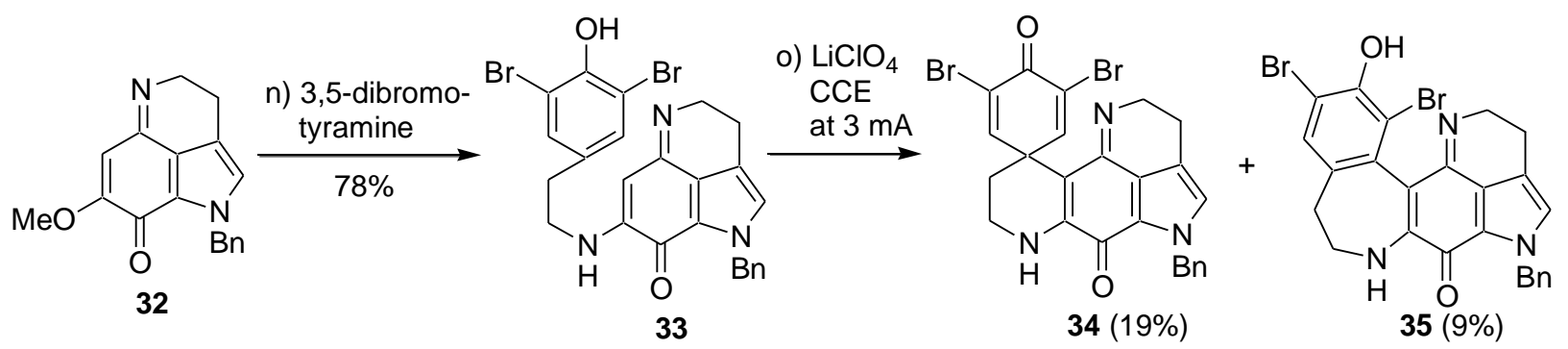

Unfortunately, in the last step, all efforts made to remove the benzyl protective group of $\mathbf{3 4}$ were unsuccessful. Therefore, they synthesized the bromophenol derivative (16). The indole (30) was hydrogenated under acidic conditions to remove the benzyl and Teoc protective groups, and the succeeding lactamization as in the case of $\mathbf{3 0}$ yielded 36. The conversion of $\mathbf{3 6}$ into $\mathbf{1 6}$ smoothly proceeded according to the procedure for the synthesis of $\mathbf{3 3}$. Upon anodic oxidation, $\mathbf{1 6}$ produced the desired discorhabdin C (7) in $24 \%$ yield accompanied by the ring-expanded product (37) in $6 \%$ yield (Scheme 5).

Scheme 5. The total synthesis of discorhabdin C by Yamamura et al.

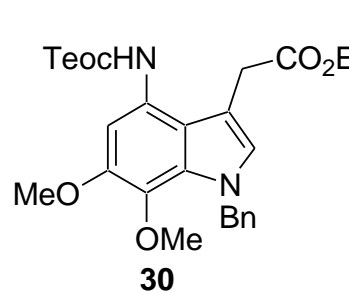

\begin{tabular}{l} 
f) $\mathrm{LiClO}_{4}, \mathrm{CCE}$ \\
at $3 \mathrm{~mA}$ \\
\hline
\end{tabular} a) $\mathrm{H}_{2}$, Pd-black $60 \% \mathrm{HClO}_{4}$

b) $\mathrm{KOH}$, then $\mathrm{DCC}$ 2 steps $36 \%$<smiles>O=C1C(Br)=CC2(C=C1Br)CCNC2=C1NCCC2=C1C1=Cc3c[nH]c(c31)C2=O</smiles>
discorhabdin C (7) $24 \%$<smiles>COc1cc2c3c(c[nH]c3c1OC)CC(=O)N2</smiles>

36<smiles>O=C1C2=C(C3=C4CNC=C4CCN13)c1c(cc(Br)c(O)c1Br)CCN2</smiles>
$37(6 \%)$<smiles></smiles>

d) CAN 3 steps $38 \%$

16 


\subsection{Heathcock's Synthesis [49]}

In 1999, Heathcock et al. accomplished the total synthesis of discorhabdin C (7) by a phenolic coupling reaction with cupric chloride. 2-Nitroguaiacol (38) was chosen as the starting material. The benzylation of the phenolic hydroxyl group and reduction of the nitro group with $\mathrm{Fe}$ and $\mathrm{HCl}$ gave the corresponding amino compound, which underwent regioselective iodination with ICl to provide the ortho-iodo derivative 39. The Heck reaction of 39 with $\mathrm{Pd}(\mathrm{OAc})_{2}$ and $(o \text {-tol })_{3} \mathrm{P}$ yielded an indole $(\mathbf{4 0})$, which was followed by reduction with $\mathrm{LiAlH}_{4}$, and protection with Boc and Ts to provide the 7-benzyloxy-1-tosyl compound (41). The debenzylation of 41 with $\mathrm{H}_{2}$ and $\mathrm{Pd} / \mathrm{C}$ afforded the 7-hydroxyindole (42), of which treatment with Fremy's salt gave the para-quinone (43). The deprotection of 43 with TFA $\left(\mathrm{CF}_{3} \mathrm{CO}_{2} \mathrm{H}\right)$ produced the indoloquinoneimine (25). The imine (25) underwent a nucleophilic substitution reaction with 3,5-dibromotyramine to furnish $\mathbf{4 4}$. The treatment of 44 with three equivalents of $\mathrm{CuCl}_{2}$ and four equivalents of $\mathrm{Et}_{3} \mathrm{~N}$ under $\mathrm{O}_{2}$ bubbling gave $\mathrm{N}$-tosyldiscorhabdin $\mathrm{C}$ (45), of which the detosylation with $\mathrm{NaOMe}$ resulted in the formation of the expected discorhabdin C (7) (Scheme 6).

Scheme 6. The total synthesis of discorhabdin C by Heathcock et al.
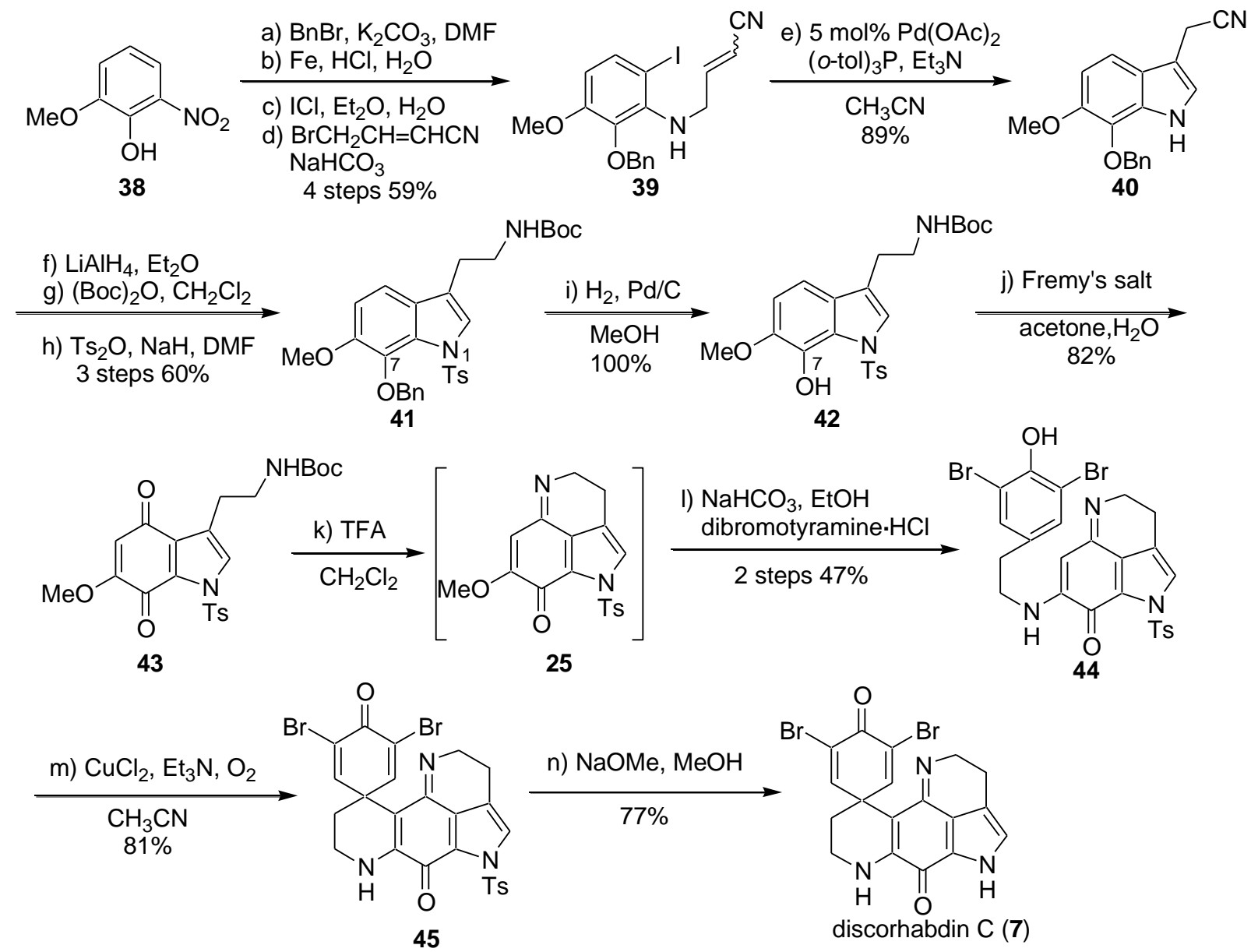

Heathcock et al. also achieved the total synthesis of ( \pm )-discorhabdin E (8) [49]. The imine (25) underwent a nucleophilic substitution reaction with $o$-bromotyramine to furnish 46. The treatment of 46 with three equivalents of $\mathrm{CuCl}_{2}$ and four equivalents of $\mathrm{Et}_{3} \mathrm{~N}$ under $\mathrm{O}_{2}$ bubbling gave 
$\mathrm{N}$-tosyldiscorhabdin $\mathrm{E}$ (47), the detosylation of which with $\mathrm{NaOMe}$ resulted in the formation of the expected ( \pm )-discorhabdin E (8).

Scheme 7. The total synthesis of ( \pm )-discorhabdin E by Heathcock et al.
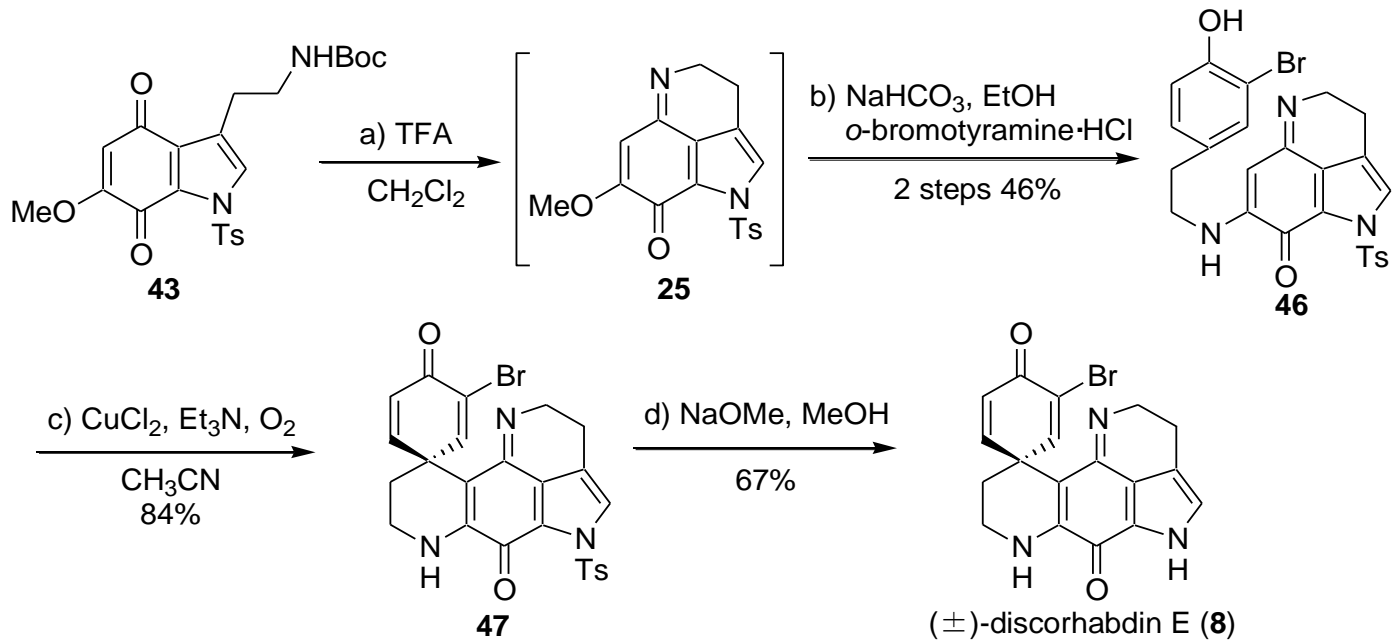

\section{Studies of the Total Synthesis of Sulfur-Containing Makaluvamine, Makaluvamine F $(48)[38,39]$}

Among the makaluvamines, makaluvamine F (48) has the most potent biological activities (e.g., cytotoxicity towards the human colon tumor cell line HCT-116 $\left(\mathrm{IC}_{50}=0.17 \mu \mathrm{M}\right)$ and inhibition of topoisomerase II). Makaluvamine F (48) has an $\alpha$-aminodihydrobenzothiophene skeleton, which is a labile $N, S$-acetal structure present in all sulfur-containing discorhabdins. Synthetic studies of the makaluvamines and discorhabdins have been carried out by several groups. However, in most cases, these efforts have been devoted only to the diverse preparations of the pyrroloiminoquinone and spirodienone units. To the best of our knowledge, the total syntheses of the sulfur-containing discorhabdins and makaluvamine $\mathrm{F}$ had not been reported until our synthesis, despite their potent cytotoxicity and their unique structure. This is probably due to the difficulty in construction of the labile and highly strained $N, S$-acetal skeletons. In our efforts to synthesize the discorhabdin alkaloids, we succeeded in the total synthesis of discorhabdin C (7) using the PIFA-mediated spirocyclization reaction. Thus, the remaining challenge for the total syntheses of these targets (the sulfur-containing family) was the construction of the labile and highly strained $N, S$-acetal skeletons. Our synthetic strategy for the total synthesis of makaluvamine F (48) is shown in Scheme 8.

Scheme 8. Retrosynthetic analysis of makaluvamine F (48).<smiles>CCCCOc1c(NC2Cc3cc(Br)c(O)cc3S2)cc2c3c(c[nH]c13)CCN2</smiles><smiles>C=CCOC(=O)O</smiles><smiles>COC1=CC2=NCCc3c[nH]c(c32)C1=O</smiles><smiles>NC1Cc2cc(Br)c(O)cc2S1</smiles> 


\subsection{Facile and Direct Synthesis of the Pyrroloiminoquinone Unit [50]}

We found that the treatment of phenyl ethers with an alkylazido group (49) in the presence of PIFATMSOTf in $\left(\mathrm{CF}_{3}\right)_{2} \mathrm{CHOH}-\mathrm{ROH}$ or $\mathrm{CF}_{3} \mathrm{CH}_{2} \mathrm{OH}-\mathrm{ROH}$ gave the corresponding quinone imine dimethylacetals (50), which in $\left(\mathrm{CF}_{3}\right)_{3} \mathrm{CHOH}-\mathrm{H}_{2} \mathrm{O}$ or $\mathrm{CF}_{3} \mathrm{CH}_{2} \mathrm{OH}-\mathrm{H}_{2} \mathrm{O}$ gave the corresponding quinine imines (51) [51] (Scheme 9). Quinone imines and quinone imine monoacetals have been proposed as the intermediates in a number of biological processes. Quinone imines were also found in the structure of marine alkaloids.

Scheme 9. Intramolecular cyclization of phenylethers bearing an alkylazido group.

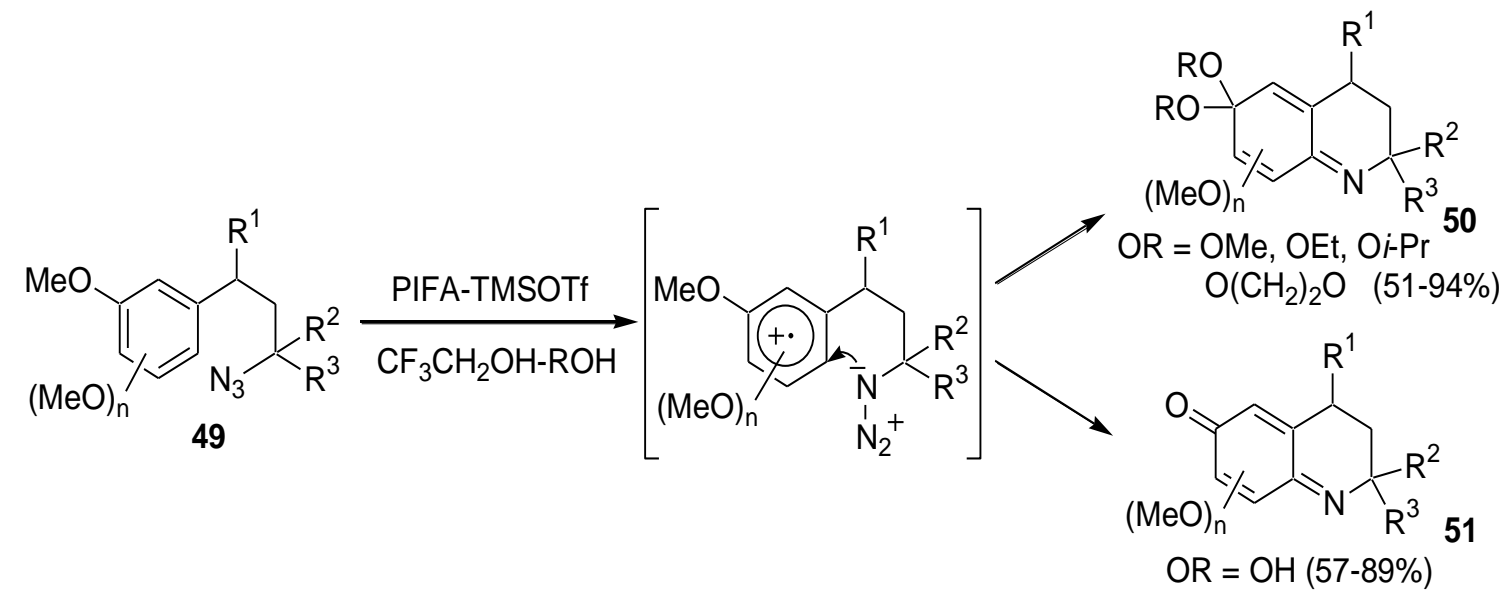

We have developed a facile and direct preparation of the quinone imine derivatives, and very recently reported the synthesis of pyrroloiminoquinones from the indole bearing an alkylazido group [52]. The starting substrate was prepared as follows. The treatment of 6,7-dimethoxyindole (52) with oxalyl chloride followed by LAH reduction gave 3-(hydroxyethyl)indole (53) (95\% yield), which was converted to 3-(azidoethyl)indole (55) via 3-(iodoethyl)indole (54) in 65\% yield. The $N$-protected 3-(azidoethyl)indoles (56a-f) were readily prepared from $\mathbf{5 5}$ by standard alkylation and acylation methods. First, we examined the reaction of $\mathbf{5 5}$ with PIFA-TMSOTf, but only a complex mixture was obtained. The reactions of $\mathbf{5 6 \mathbf { a }}(\mathrm{R}=\mathrm{Me})$ and $\mathbf{5 6} \mathbf{b}\left(\mathrm{R}=\mathrm{Si}(i-\mathrm{Pr})_{3}\right)$ with PIFA-Me $\mathrm{S}_{3} \mathrm{SiOTf}$ also gave complex mixtures, probably due to the side reactions occurring at the 1- or 3-position on the indole nucleus. In fact, several groups have already reported that a 1-, 2- or 3-substituted indole derivative is obtained from the reaction of indoles with iodobenzene diacetate. Therefore, we examined the reactions of the 1-protected indoles (56c-f) with PIFA-Me $\mathrm{SSiOTf}_{3}$ in the presence of $\mathrm{H}_{2} \mathrm{O}$. We found that the corresponding $N$-deprotected pyrroloiminoquinones (57) or the $N$-protected pyrroloiminoquinones (25 and $\mathbf{5 8}$ ) were obtained in moderate yields when the indoles protected with an electron-withdrawing group, such as the acetyl, benzoyl, tosyl or benzyloxycarbonyl group, were treated with PIFA-Me $\mathrm{SiOTf}_{3}$ in $\left(\mathrm{CF}_{3}\right)_{2} \mathrm{CHOH}-\mathrm{H}_{2} \mathrm{O}$ (50:1) (Scheme 10). 
Scheme 10. A novel synthetic method for pyrroloiminoquinones.

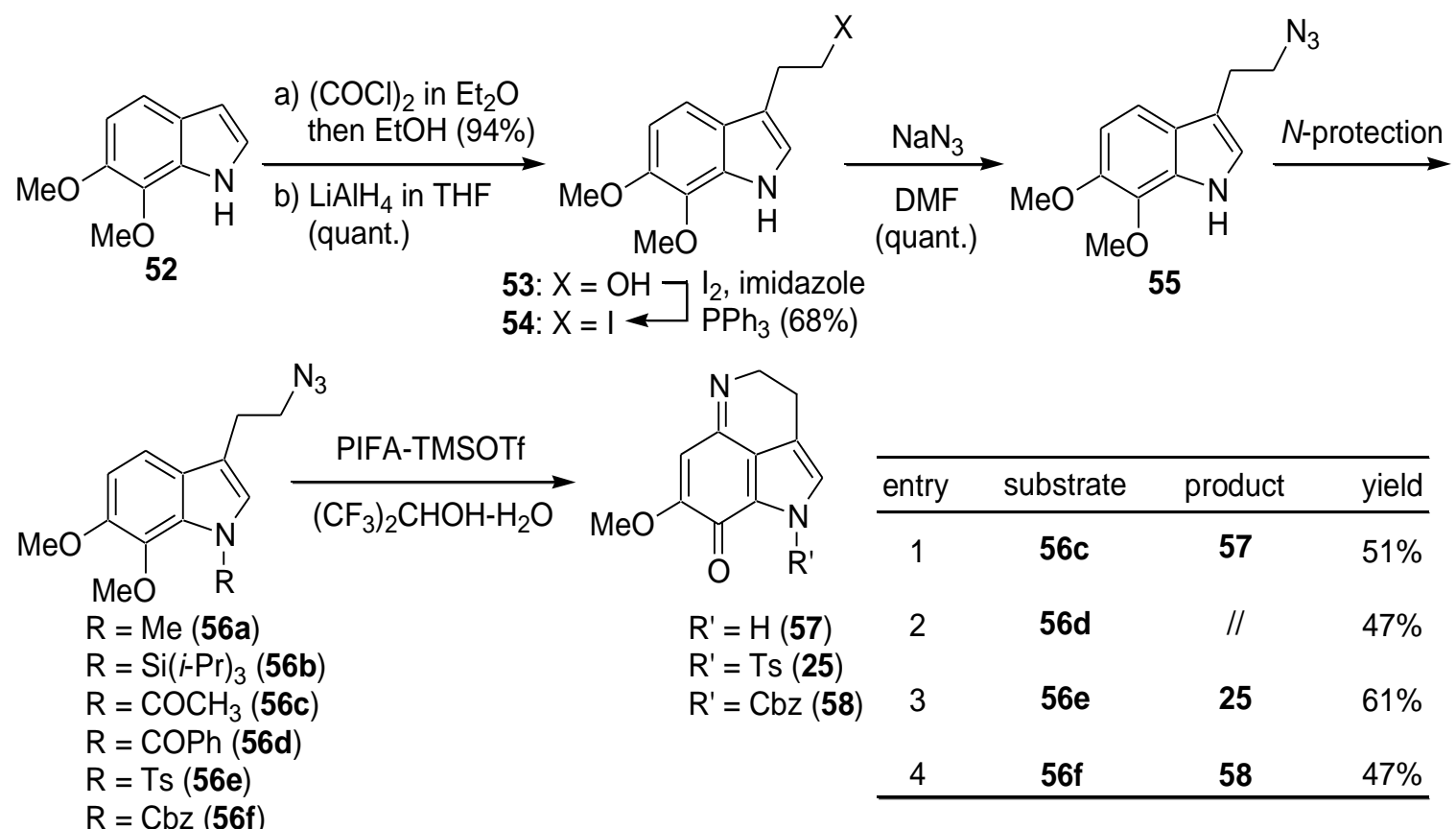

$N$-protection: 56c; AcCl, $t$-BuOK in THF (91\%). 56d; BzCl, $t$-BuOK in THF (quant). 56e; TsCl, $t$-BuOK in THF (89\%). 56f; $\mathrm{ClCO}_{2} \mathrm{Bn}, t$-BuOK in THF (96\%)

\subsection{Synthesis of the Dihydrothiophene Units [52,53]}

In our previous studies, we developed the direct sulfenylation of phenyl ethers using PIFA [54,55]. Furthermore, we developed the intramolecular cyclization of phenyl ethers (59) bearing an alkylsulfide group using PIFA in $\left(\mathrm{CF}_{3}\right)_{2} \mathrm{CHOH}$ and PIFA- $\mathrm{BF}_{3} \cdot \mathrm{Et}_{2} \mathrm{O}$ in $\mathrm{CH}_{2} \mathrm{Cl}_{2}$ that specifically produced the corresponding sulfur-containing heterocycles (60) (Scheme 11) [52].

Scheme 11. Intramolecular cyclization of phenylethers bearing an alkyl sulfide group.

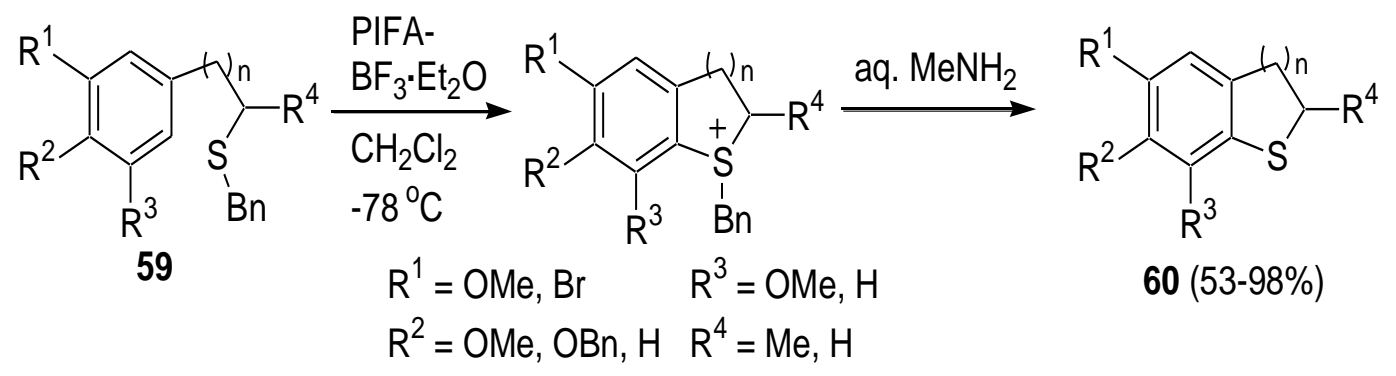

A plausible reaction mechanism is that the electron-rich aromatic ring is initially oxidized by the activated PIFA via a single electron transfer (SET), and then the sidechain attacks the radical cation. Although other mechanisms, such as the one via the sulfonium salt could also be possible, we proposed the mechanism via the radical cation (Scheme 12). 
Scheme 12. A plausible mechanism for the cyclization reaction of $\mathbf{5 9}$.

$$
\left[\begin{array}{c}
\mathrm{R}_{\mathrm{B}} \\
\text { cation radical }
\end{array}\right]
$$

We constructed the dihydrobenzothiophenes using the direct sulfenylation of phenol ethers with PIFA and arylthiols. The synthesis was initiated from the methyl 4-hydroxyphenylacetate (61) to afford the phenol ether (63) bearing an alkyl sulfide side chain in five steps. The treatment of the phenol ether $(\mathbf{6 3})$ with PIFA- $\mathrm{BF}_{3} \cdot \mathrm{Et}_{2} \mathrm{O}$ followed by the treatment of aqueous $\mathrm{MeNH}_{2}$ provided the dihydrobenzothiophenes (64) (Scheme 13).

Scheme 13. Synthesis of dihydrobenzothiophene.

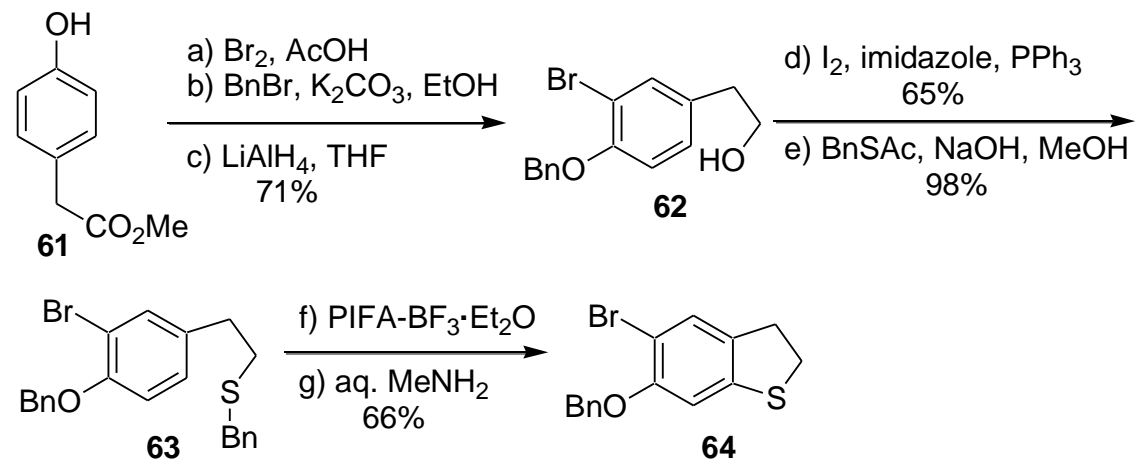

Next, we attempted to synthesize the $\alpha$-azidodihydrobenzothiophenes, which can serve as an $N, S$ acetal equivalent, by the introduction of an azido group to the dihydrobenzothiophene derivatives. After considerable effort, we found a novel and direct $\alpha$-azidation method for cyclic sulfides using a combination of $\mathrm{PhI}=\mathrm{O}$ and $\mathrm{Me}_{3} \mathrm{SiN}_{3}$ (Scheme 14) [53]. A plausible reaction mechanism is proposed in Scheme 15 . The sulfonium cation (66) initially formed by the reaction of the sulfide with $\mathrm{PhI}=\mathrm{O}$ $\mathrm{Me}_{3} \mathrm{SiN}_{3}$, whose combination was studied by Magnus and co-workers, is then deprotonated to give a cation intermediate (67). The azido anion attacks the $\alpha$-position of 67 to produce the $\alpha$-azido sulfide (65).

Scheme 14. Synthesis of $\alpha$-azidodihydrobenzothiophene derivatives.

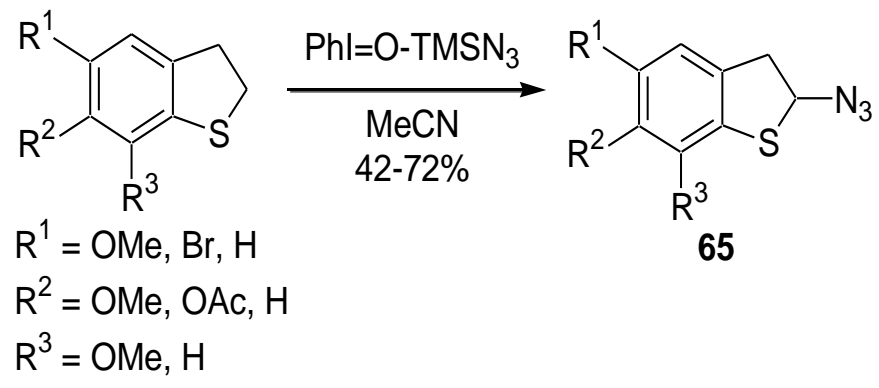


Scheme 15. A plausible reaction mechanism for $\alpha$-azidation of dihydrobenzothiophene derivatives.

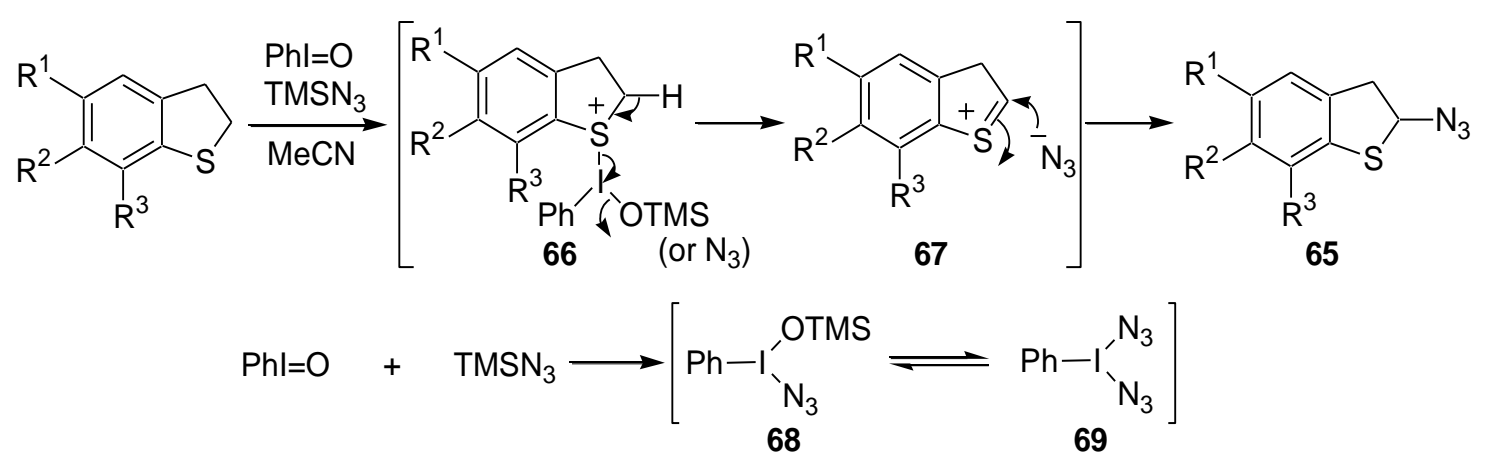

Although azidation of 5-bromo-6-benzyloxydihydrobenzothiophene (70) gave only a trace amount of the expected $\alpha$-azido compound, treatment of the acetylated compound (71) with $\mathrm{PhI}=\mathrm{O}$ and $\mathrm{Me}_{3} \mathrm{SiN}_{3}$ followed by hydrolytic deprotection of the 6-acetoxy group provided the 2-azido-5-bromo-6hydroxydihydrobenzothiophene (72) (Scheme 16).

Scheme 16. The synthesis of 2-azido-5-bromo-6-hydroxydihydrobenzothiophene (72).
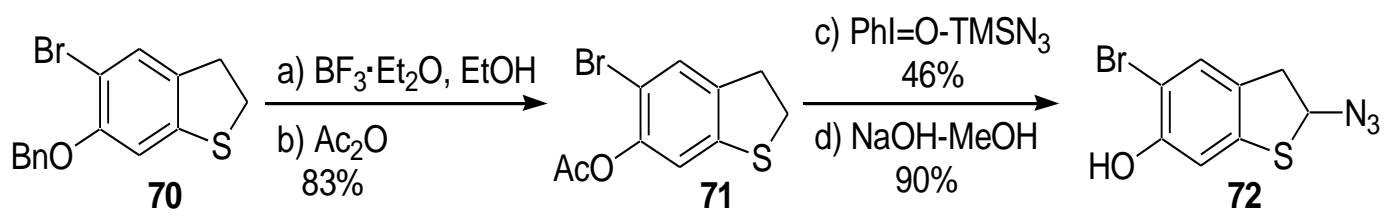

\subsection{Total Synthesis of $( \pm)$-Makaluvamine F (48) $[38,39]$}

Catalytic hydrogenation of $\mathbf{7 2}$ followed by the coupling reaction with $\mathbf{5 7}$ in $\mathrm{MeOH}$ in the presence of $\mathrm{Me}_{3} \mathrm{SiOTf}$ gave makaluvamine $\mathrm{F}$ only in a poor yield. Finally, we found that the catalytic hydrogenation of $\mathbf{7 2}$ using 10\% Pd-C in the presence of four equivalents of trifluoroacetic acid (TFA) resulted in complete reduction leading to a TFA salt in quantitative yield without any side reactions. The final coupling reaction in $\mathrm{MeOH}$ between both synthetic precursors, the TFA salt and 57, proceeded to give the TFA salt of makaluvamine F (48) (Scheme 17).

Scheme 17. The total synthesis of ( \pm )-makaluvamine F (48).

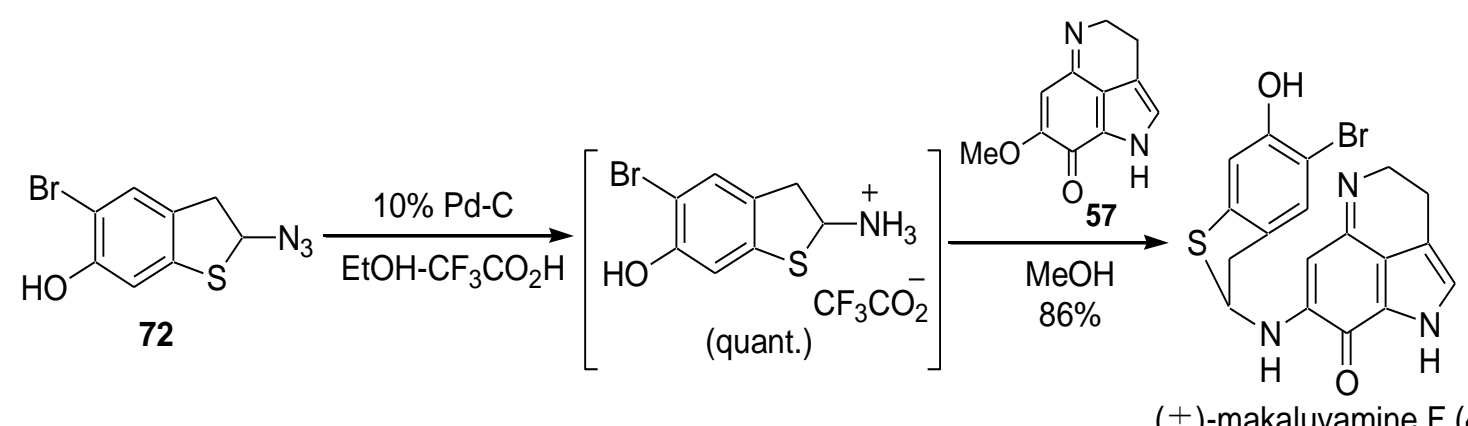

$( \pm)$-makaluvamine $\mathrm{F}(\mathbf{4 8})$ 


\section{Total Synthesis of Discorhabdin A [40-43]}

In 1987, prianosin A [28] was isolated from the Okinawan sponge Prianos melanos by Kobayashi et al., and in 1988, discorhabdin A [29] was isolated from New Zealand sponges of the genus Latrunculia by Munro et al. Some years later, it was found that prianosin A and discorhabdin A were the same compound. Discorhabdin A (prianosin A) is antimicrobial and has a strong cytotoxicity ( $\mathrm{IC}_{50}$ values of $37 \mathrm{ng} / \mathrm{mL}, 14 \mathrm{ng} / \mathrm{mL}, 40 \mathrm{ng} / \mathrm{mL}$ and $13 \mathrm{ng} / \mathrm{mL}$ against L1210, L5178Y, P388 and xrs-6 in vitro, respectively, and $\mathrm{ED}_{50}$ values of $50 \mathrm{ng} / \mathrm{mL}$ against P388). Our next synthetic target was discorhabdin A (1), which has a unique sulfur-containing fused ring system incorporating azacarbocyclic spirocyclohexanone and pyrroloiminoquinone systems, and shows the most powerful cytotoxic activity among the isolated discorhabdins.

First, on the basis of the hypothesis by Munro and co-workers [56], we examined the biosynthetically plausible route from makaluvamine F (48) using our previously developed oxidative spirocyclization reaction with PIFA. As a result, the oxidative cyclization of makaluvamine F (48) as well as the trimethylsilylated makaluvamine F using PIFA under various conditions yielded a complex mixture, probably due to the high reactivity of the iodine(III) reagent toward the sulfide group, the aminoiminoquinone skeleton, and the phenolic $\mathrm{OH}$ group in makaluvamine $\mathrm{F}$ (48) (Scheme 18).

Scheme 18. Synthetic approach of discorhabdin A from makaluvamine F.
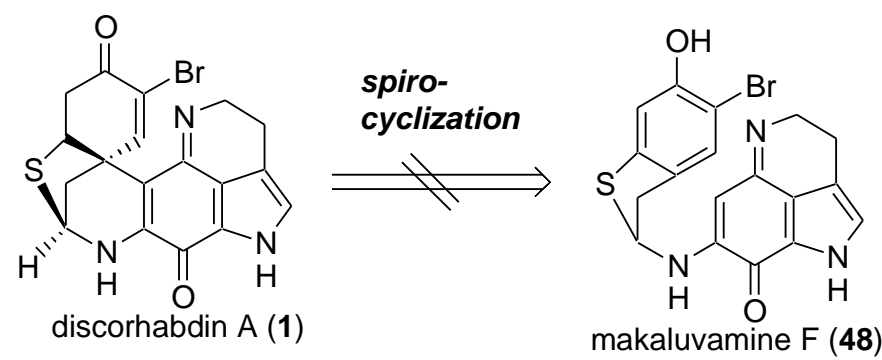

Therefore, we altered the synthetic strategy. The new strategy included the pre-construction of the spirodienone system using the hypervalent iodine(III) reagent and the final introduction of the sulfur group to the cross-linked system. We first examined the shorter path via an amino acid derivative. The tritylation of the L-tyrosine methyl ester hydrochloride (73) with $\operatorname{TrCl}$ and $\mathrm{Et}_{3} \mathrm{~N}$ followed by monobromination with NBS yielded $\mathbf{7 4}$ (two steps, 65\% yield). The coupling reaction of $\mathbf{7 4}$ with pyrroloiminoquinone 25, which was prepared by our previously developed PIFA-induced pyrroloiminoquinone formation, provided 75 as its $\mathrm{CF}_{3} \mathrm{CO}_{2} \mathrm{H}$ salt (75.TFA). We then examined the oxidative spirocyclization reaction of 75.TFA using PIFA. Although the various reaction conditions tested, this reaction did not give the corresponding spirodienone, but yielded a complex mixture; probably because generation of the free base of $\mathbf{7 5}$ was difficult. This might be because 75.TFA has an active methine proton and readily decomposed under basic conditions (even in the presence of $\mathrm{Et}_{3} \mathrm{~N}$ ). Thus, we modified the synthetic strategy using an alternative route via the amino alcohol as follows: reduction of $\mathbf{7 4}$ with DIBAH followed by silylation of the resulting alcohol with TBSCl gave the bissilylated compound 76, and a coupling reaction with pyrroloiminoquinone 25 yielded 77. The spirodienone formation of $\mathbf{7 7}$ using PIFA effectively proceeded in the presence of MK10 to give a 
diastereomeric mixture of $\mathbf{7 8}$ and $\mathbf{7 8}$ '. Both diastereomers were readily separated by column chromatography on silica gel to give the less polar isomer $\mathbf{7 8}$ and the polar isomer 78' (Scheme 19).

Scheme 19. The synthesis of spirodienone.

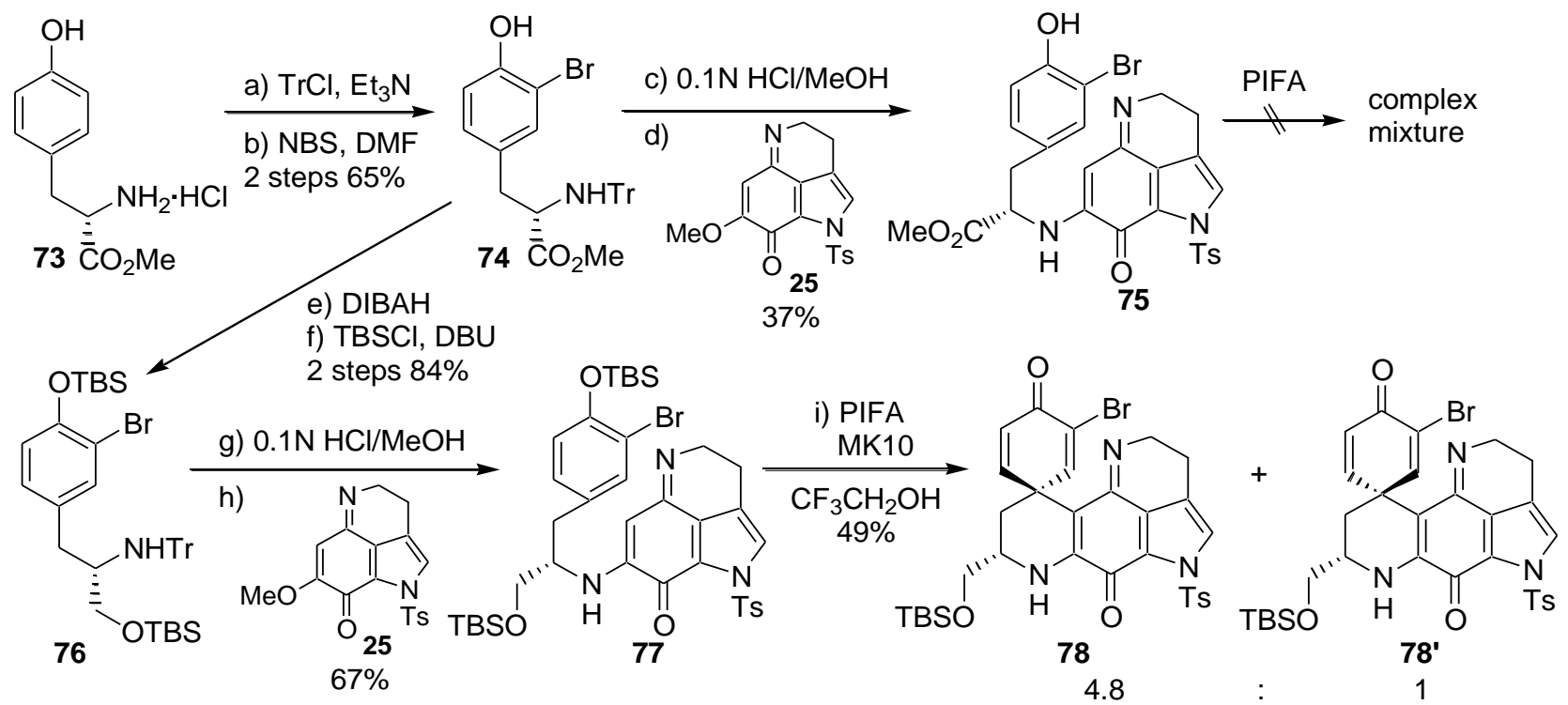

The major isomer 78, whose absolute stereochemistry ( $S$ configuration) of the spirocenter (C-6) is the same as that of natural discorhabdin $\mathrm{A}(\mathbf{1})$, was desilylated by $\mathrm{BF}_{3} \cdot \mathrm{Et}_{2} \mathrm{O}$, and then converted into the $\mathrm{N}, \mathrm{O}$-acetal intermediate 79 by an oxidative fragmentation reaction with $\mathrm{C}_{6} \mathrm{~F}_{5} \mathrm{I}\left(\mathrm{OCOCF}_{3}\right)_{2}$ and $\mathrm{MeOH}$. The $p$-methoxybenzylthio group was efficiently introduced in the presence of $\mathrm{BF}_{3} \cdot \mathrm{Et}_{2} \mathrm{O}$ to give the unstable $N, S$-acetal $\mathbf{A}$ as a diastereomeric mixture. The debenzylation of $\mathbf{A}$, a labile and highly functionalized compound, also required the mildest possible reaction conditions. Our initial strategy was to perform a mild debenzylation on the $p$-methoxybenzylsulfonium salt formed by the 1,4 -addition of a sulfide group to the cyclohexadienone moiety. Accordingly, we treated a diastereomeric mixture of A with $30 \% \mathrm{HBr}-\mathrm{AcOH}$ followed by aqueous work up with $\mathrm{MeNH}_{2}$ that produced the $\mathrm{N}$-tosylated discorhabdin A (80). Ultimately, we developed an efficient one-pot transformation procedure yielding 80 from 79. The procedure using $p$-methoxybenzylthiol in $30 \% \mathrm{HBr}-\mathrm{AcOH}\left(36 \mathrm{~h} ;-78 \sim 4{ }^{\circ} \mathrm{C}\right)$ followed by treatment with aqueous $\mathrm{MeNH}_{2}$ mainly gave 80: the enantiomeric excess (ee) of $\mathbf{8 0}$ was confirmed to be $>99 \%$ by HPLC analysis using a chiral column (DAICEL CHIRALCEL OD; $n$-Hex $/ i$-PrOH = $65 / 35 ; 20^{\circ} \mathrm{C}$ ). Finally, removal of the tosyl group of $\mathbf{8 0}$ with NaOMe in THF gave discorhabdin A (1) in the optically pure form for the first time (Scheme 20. The synthetic product as its $\mathrm{HCl}$ salt was identical in all respects with the natural discorhabdin A including its optical rotation ssynthetic discorhabdin $\mathrm{A}:[\alpha]_{\mathrm{D}}+388(\mathrm{c}=0.06, \mathrm{MeOH})$; natural discorhabdin $\left.\mathrm{A}:[\alpha]_{\mathrm{D}}+400(\mathrm{c}=0.05, \mathrm{MeOH})\right\}$ and ${ }^{1} \mathrm{H}$ NMR nOe data. 
Scheme 20. The total synthesis of (+)-discorhabdin A.
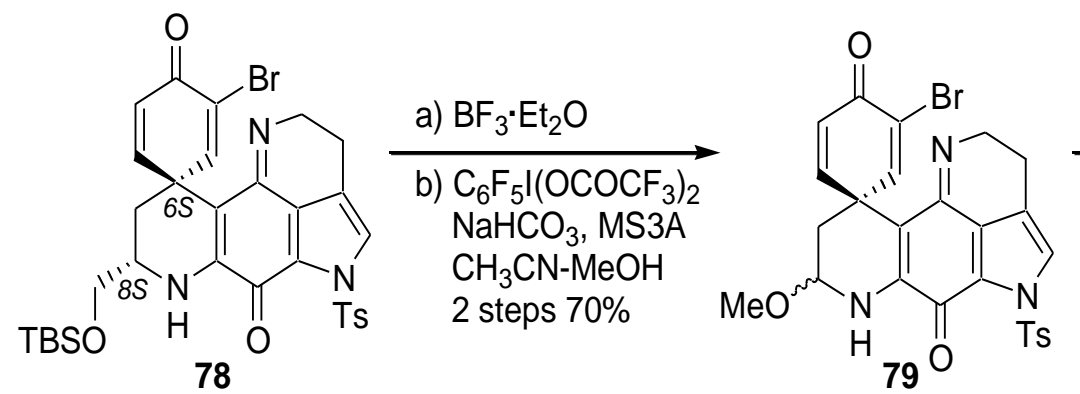

$$
\begin{aligned}
& \text { c) } p-\mathrm{MeOBnSH} \\
& \underset{-78 \sim 4{ }^{\circ} \mathrm{C}}{\stackrel{\mathrm{HBr}-\mathrm{AcOH}}{\mathrm{CH}_{2} \mathrm{Cl}_{2}}}
\end{aligned}
$$
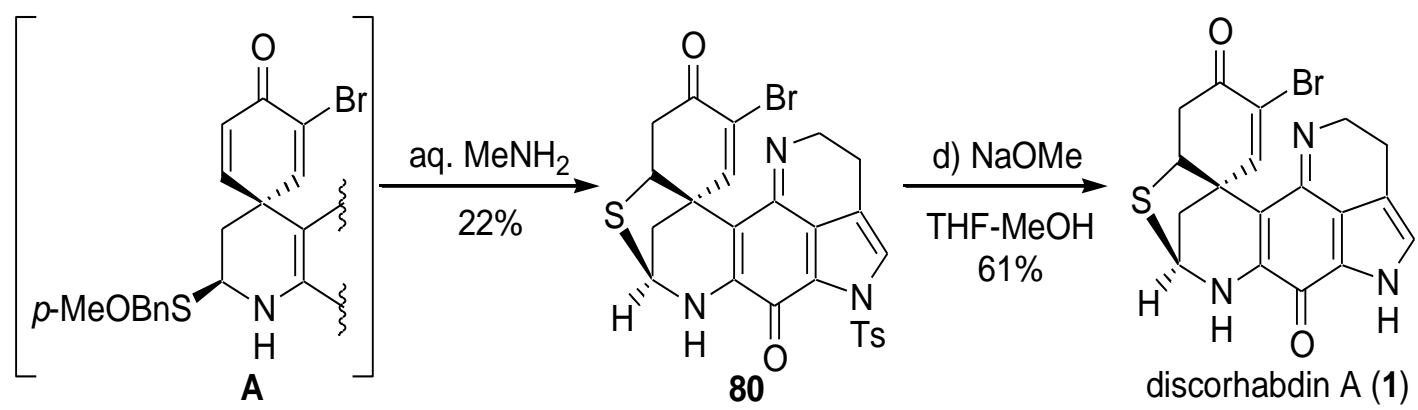

Furthermore, we also completed the total synthesis of the unnatural discorhabdin A, (-)discorhabdin A, starting from the D-tyrosine methyl ester by the same route as described for $(+)$ discorhabdin A (1) \{synthetic product: $\left.[\alpha]_{\mathrm{D}}-390(\mathrm{c}=0.05, \mathrm{MeOH})\right\}$.

\section{Semi-Synthesis of Discorhabdins P and U [57]}

In 2006, Copp et al. reported the semi-synthetic preparation of discorhabdins $\mathrm{P}(\mathbf{8 1})$ and $\mathrm{U}(\mathbf{1 3})$ from natural discorhabdins C (7) and B (4). In New Zealand waters, Latrunculia sp. sponges are a rich source of the discorhabdin alkaloids, with organisms capable of producing either discorhabdins A (1), $\mathrm{B}$ (4) or C (7) as their major secondary metabolites, typically in isolated yields of $25-35 \mathrm{mg} / \mathrm{g}$ dry sponge weight [58]. The aim of the Copp et al. study was to investigate the methylation of discorhabdin $\mathrm{C}(\mathbf{7})$ in an effort to prepare discorhabdin $\mathrm{P}(\mathbf{8 1})$ or related $N$-methyl analogs for further biological testing, and for the first time, to prepare similar analogs of discorhabdin B (4) in an effort to expand the structure-activity relationship understanding of this class of compounds. Discorhabdin $\mathrm{C}$ (7) was reacted with $\mathrm{CH}_{3} \mathrm{I}$ in dry acetone to yield a single major product, the 13- $N$-methyl analog discorhabdin $\mathrm{P}(\mathbf{8 1})$ in $54 \%$ yield (Scheme 21). Discorhabdin B (4) was reacted with $\mathrm{CH}_{3} \mathrm{I}$ in dry acetone to yield two products, discorhabdin U (13) and 82. The products of this reaction were dependent upon the molar equivalents of $\mathrm{CH}_{3} \mathrm{I}$ used: with a large excess (10-25 equivalent) yielding predominantly discorhabdin $\mathrm{U}(\mathbf{1 3})$, while a two molar equivalent of $\mathrm{CH}_{3} \mathrm{I}$ yielded the mono-methyl $\mathbf{8 2}$. The order of the methylation of discorhabdin B (4) preferentially favors the thio group and a large excess of $\mathrm{CH}_{3}$ I enables the methylation at the $\mathrm{N}-13$ pyrrole position. 
Scheme 21. The semi-synthesis of discorhabdin P and U.<smiles></smiles>

discorhabdin C (7)

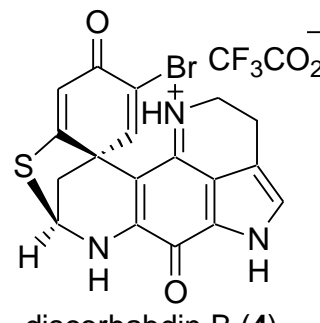

discorhabdin B (4)

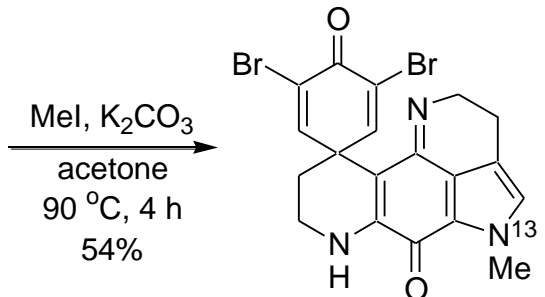

discorhabdin $\mathrm{P}(\mathbf{8 1})$<smiles></smiles>

$(39 \%)$

\section{Total Synthesis of Prianosin B [44]}

Although, some 20 years ago, Kobayashi et al. also reported one such family, prianosin B (2), which has the 16,17-dehydropyrroloiminoquinone structure, its synthesis has not yet been reported [17]. For the synthesis of prianosin B (2), the construction of the 16,17-dehydropyrroloiminoquinone structure is an important issue. We have succeeded in the first total synthesis of the sulfur-containing discorhabdin, discorhabdin A [40-43]. We postulated that the prianosins and discorhabdins with the 16,17-dehydropyrroloiminoquinone moiety can be synthesized by dehydrogenation of the pyrroloiminoquinone of the discorhabdin A (1) or discorhabdin A intermediate (Scheme 22).

Scheme 22. Synthetic strategy for prianosin B.

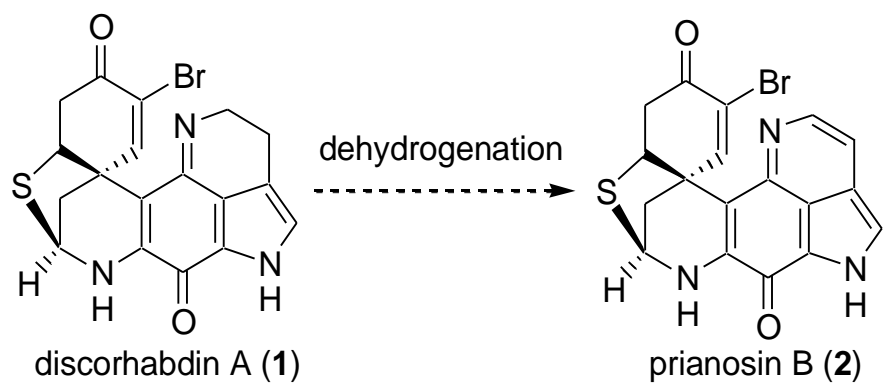

We first examined the dehydrogenation reaction of the simple spirodienone (83) with various oxidants as the model reaction. However, reactions with oxidants, such as DDQ, CAN, $\mathrm{MnO}_{2}$ and $\mathrm{Pd} / \mathrm{C}$ (Yamamura's conditions) [59], gave poor results (Scheme 23). 
Scheme 23. Model study using various oxidants.<smiles>O=C1C=CC2(C=N1)CCNC2=C1C(=O)c2c3c(cn21)CCN3</smiles>

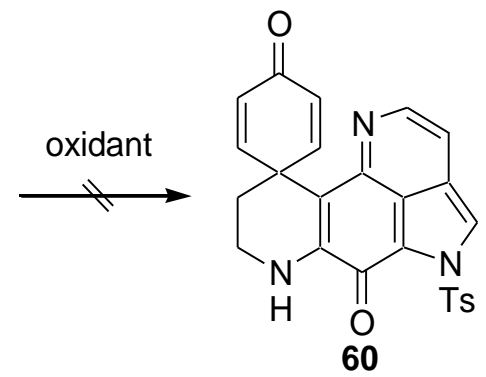

We next tried the use of nucleophiles, because White et al. reported that the dehydrogenation and detosylation of the pyrroloiminoquinone system using sodium azide as a nucleophile [60]. From a detailed study of nucleophiles, we found that the use of a catalytic amount of $\mathrm{NaN}_{3}$ produced the best result. Since it was revealed that the detosylation and dehydrogenation reaction of the pyrroloiminoquinone unit with a catalytic amount of $\mathrm{NaN}_{3}$ proceeded in good yield, the total synthesis of prianosin B (2) was next studied (Scheme 24). In our own total synthesis of discorhabdin A (1), the $\mathrm{N}, \mathrm{O}$-acetal intermediate (79) was prepared from the L-tyrosine methyl ester hydrochloride in eight steps. The sulfur cross linkage reaction of the intermediate was carried out using $p$-MeOBnSH and $30 \% \mathrm{HBr}-\mathrm{AcOH}$ followed by aqueous $\mathrm{MeNH}_{2}$ to give the sulfur-linked pyrroloiminoquinone compound 80. The treatment of $\mathbf{8 0}$ with $\mathrm{NaN}_{3}$ in DMF caused detosylation and dehydrogenation to produce prianosin B (2) in $48 \%$ yield. The spectral data were identical to that reported for the natural prianosin B (2) $\left([\alpha]^{23}{ }_{\mathrm{D}}+362\left(\mathrm{c} 0.405, \mathrm{CHCl}_{3}\right)\right.$, lit. $\left.[\alpha]^{30}{ }_{\mathrm{D}}+360\left(\mathrm{c} 0.1, \mathrm{CHCl}_{3}\right)[17]\right)$.

Scheme 24. The total synthesis of prianosin B.
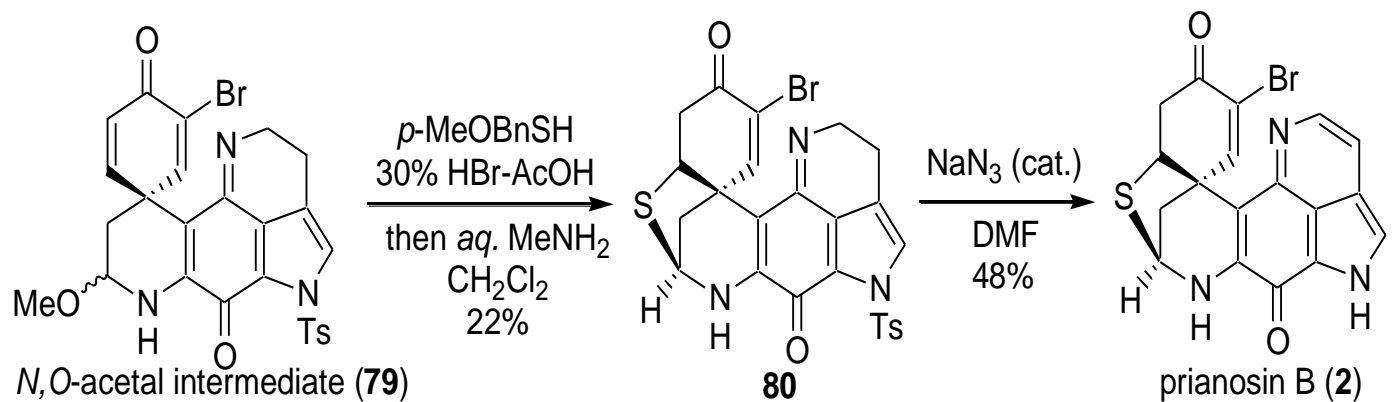

For determining the reaction mechanism, two studies were carried out. One was the measurement of the mass spectrum of the reaction mixture. A suspension of spirodienone $\mathbf{8 3}$ and $\mathrm{NaN}_{3}$ in $\mathrm{DMF}$ was stirred under nitrogen at $70{ }^{\circ} \mathrm{C}$ for $40 \mathrm{~min}$. The mass spectrum of the reaction mixture revealed the ion peak of $\mathrm{TsN}_{3}$. The other was the reaction of the $\mathrm{N}-\mathrm{H}$ spirodienone 85 and $\mathrm{TsN}_{3}$ under basic conditions. Thus, $\mathrm{NaH}$ and $\mathrm{TsN}_{3}$ were added to a solution of $\mathbf{8 5}$ in DMF and the aromatic compound $\mathbf{8 6}$ was obtained in $41 \%$ yield (Scheme 25 ). 
Scheme 25. The reaction of $N-\mathrm{H}$ spirodienone, $\mathrm{TsN}_{3}$ and $\mathrm{NaH}$.

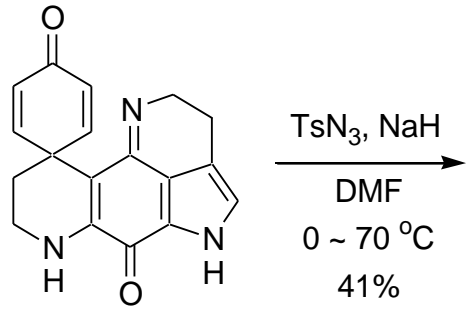

85

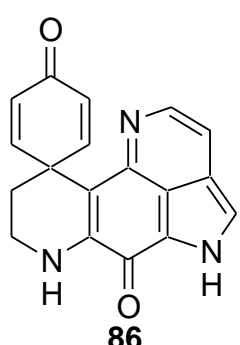

86

A plausible reaction mechanism for the present dehydrogenation reaction of pyrroloiminoquinone using $\mathrm{NaN}_{3}$ is illustrated in Scheme 26. It would involve the nucleophilic attack of $\mathrm{N}_{3}{ }^{-}$on the tosyl residue followed by the addition of metalo enamine to $\mathrm{TsN}_{3}$ to produce the intermediate $\mathbf{I}$ [61-63] Dehydrogenation would proceed by the intramolecular elimination via a six-membered transition state to produce the intermediate III and isomerization to produce 86 [64-65]. Reproduction of the azide anion during the reaction would make possible the use of the catalytic amount of $\mathrm{NaN}_{3}$. The reproduction of the azide anion was deduced from the presence of toluenesulfinic acid observed in the ${ }^{1}$ H-NMR spectrum (Scheme 26).

Scheme 26. A plausible mechanism for the dehydrogenation reaction of pyrroloiminoquinone using $\mathrm{NaN}_{3}$.

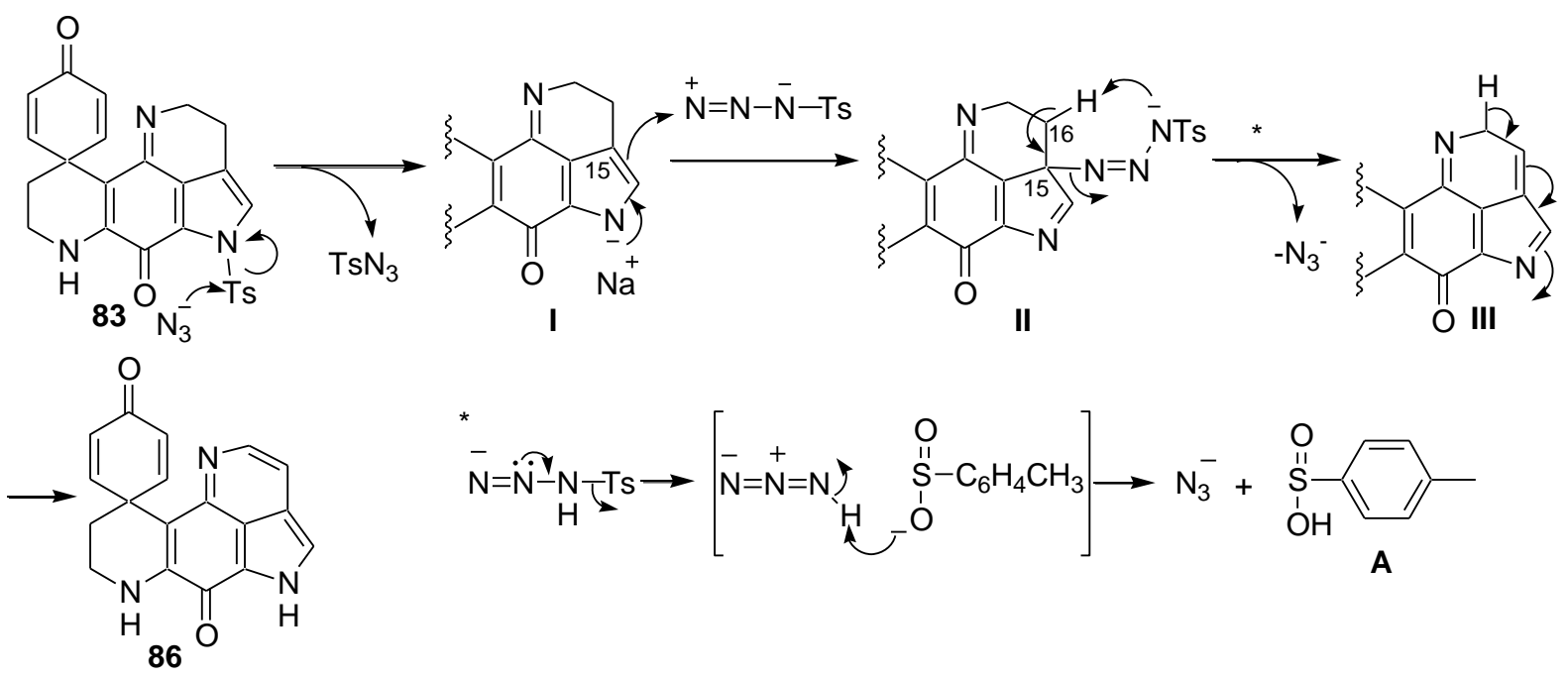

\section{Summary}

In this review, we described the existing studies on the total synthesis of the discorhabdins. Because of their prominent potent antitumor activity and unusual ring structure, synthesis of the discorhabdins has attracted the interests of many organic chemists. Many synthetic approaches have appeared over the past decade, however, only few discorhabdins have been completely synthesized. We have accomplished the total syntheses of discorhabdins C, A and prianosin B. Discorhabdin A has the strongest activity in vitro, but no activity in vivo. Therefore, we are synthesizing a variety of discorhabdin analogs [66]. Although many synthetic studies of the discorhabdin alkaloids have already appeared, biological studies including structure activity relationship or the mode of action of the 
discorhabdins have not yet significantly occurred [67-71]. In the next stage, it is necessary to study the structure activity relationship and the mode of action of the discorhabdins.

\section{References and Notes}

1. Carté, B.K. Biomedical potential of marine natural products. BioScience 1996, 46, 271-285.

2. Faulkner, D.J. Biomedical Uses for Natural Marine Chemicals. Oceanus 1992, 35, $29-35$.

3. Faulkner, D.J. Marine natural products: metabolites of marine algae and herbivorous marine mollusks. Nat. Prod. Rep. 1984, 1, 251-280.

4. Barasch, D. Fishing for Drugs. Am. Health 1990, 9, 20-21.

5. Cohen, T. Pharmaceuticals from the sea. Technol. Rev. 1993, 96, 15-16.

6. Liu, R.; Cui, C.; Duan, L.; Gu, Q.; Zhu, W. Potent in vitro anticancer activity of metacycloprodigiosin and undecylprodigiosin from a sponge-derived actinomycete Saccharopolyspora sp. nov. Archives of Pharm. Res. 2005, 28, 1341-1344.

7. Jeong, S.Y.; Ishida, K.; Ito, Y.; Okada, S.; Murakami, M. Bacillamide, a novel algicide from the marine bacterium, Bacillus sp. SY-1, against the harmful dinoflagellate, Cochlodinium polykrikoides. Tetrahedron Lett. 2003, 44, 8005-8007.

8. Xin, Z.H.; Zhu, W.M.; Gu, Q.Q.; Fang, Y.C.; Duan, L.; Cui, C.B. A new cytotoxic compound from Penicillium auratiogriseum, symbiotic or epiphytic fungus of sponge Mycale plumose. Chin. Chem. Lett. 2005, 16, 1227-1229.

9. Xin, Z.; Tian, L.; Zhu, T.; Wang, W.; Du, L.; Fang, Y.; Gu, Q.; Zhu, W. Isocoumarin derivatives from the sea squirt-derived fungus Penicillium stoloniferum QY2-10 and the halotolerant fungus Penicillium notatum B-52. Arch. Pharm. Res. 2007, 30, 816-819.

10. Wang, F.; Fang, Y.; Zhang, M.; Lin, A.; Zhu, T.; Gu, Q.; Zhu, W. Six new ergosterols from the marine-derived fungus Rhizopus sp. Steroids 2008, 73, 19-26.

11. Wang, F.; Fang, Y.; Zhu, T.; Zhang, M.; Lin, A.; Gu, Q.; Zhu, W. Seven new prenylated indole diketopiperazine alkaloids from holothurianderived fungus Aspergillus fumigatus. Tetrahedron 2008, 64, 7986-7991.

12. Urban, S.; Hickford, S.J.H.; Blunt, J.W.; Munro, M.H.G. Bioactive Marine Alkaloids. Curr. Org. Chem. 2000, 4, 765-805.

13. Gunasekera, S.P.; Zuleta, I.A.; Longley, R.E.; Wright, A.E.; Pomponi, S.A. Discorhabdins S, T, and U, New Cytotoxic Pyrroloiminoquinones from a Deep-Water Caribbean Sponge of the Genus Batzella. J. Nat. Prod. 2003, 66, 1615-1617.

14. Reyes, F.; Martin, R.; Rueda, A.; Fernandez, R.; Montalvo, D.; Gomez, C.; Sanchez-Puelles, J.M. Discorhabdins I and L, Cytotoxic Alkaloids from the Sponge Latrunculia brevis. J. Nat. Prod. 2004, 67, 463-465.

15. Antunes, E.M.; Beukes, D.R.; Kelly, M.; Samaai, T.; Barrows, L.R.; Marshall, K.M.; Sincich, C.; Davies-Coleman, M.T. Cytotoxic Pyrroloiminoquinones from Four New Species of South African Latrunculid Sponges. J. Nat. Prod. 2004, 67, 1268-1276.

16. Harayama, Y.; Kita, Y. Pyrroloiminoquinone alkaloids. Discorhabdins and makaluvamines. Curr. Org. Chem. 2005, 9, 1567-1588. 
17. Cheng, J.; Ohizumi, Y.; Wälchli, M.R.; Nakamura, H.; Hirata, Y.; Sasaki, T.; Kobayashi, J. Prianosins B, C, and D, novel sulfur-containing alkaloids with potent antineoplastic activity from the Okinawan marine sponge Prianos melanos. J. Org. Chem. 1988, 53, 4621-4624.

18. Monks, A.; Scuderio, D.A.; Skehan, P.; Shoemaker, R.H.; Paull, K.D.; Hose, V.C.; Langley, J.; Cronise, P.; Vaigro-Wolff, A.; Grey-Goodrich, M.; Campball, H.; Boyd, M.R. Feasibility of a high-flux anticancer drug screen using a diverse panel of cultured human tumor cell lines. J. Natl. Cancer Inst. 1991, 83, 757-766.

19. Carney, J.R.; Scheuer P.J.; Kelly-Borges, M. Makaluvamine G, a cytotoxic pigment from an an Indonesian Sponge Histodermella sp. Tetrahedron 1993, 49, 8483-8486.

20. Schmidt, E.W.; Harper, M.K.; Faulkner, D.J. Makaluvamines H-M and Damirone C from the Pohnpeian Sponge Zyzzya fuliginosa. J. Nat. Prod. 1995, 58, 1861-1867.

21. Venables, D.A.; Concepción, G.P.; Matsumoto, S.; Barrows, L.R.; Ireland, C.M. Makaluvamine N: A New Pyrroloiminoquinone from Zyzzya fuliginosa. J. Nat. Prod. 1997, 60, 408-410.

22. Sun, H.H.; Sakemi, S.; Burres, N.; McCarthy, P. Isobatzellines A, B, C, and D. Cytotoxic and antifungal pyrroloquinoline alkaloids from the marine sponge Batzella sp. J. Org. Chem. 1990, $55,4964-4966$.

23. Gunasekera, S.P.; McCarthy, P.J.; Longley, R.E.; Pomponi, S.A.; Wright, A.E. Secobatzellines A and B, Two New Enzyme Inhibitors from a Deep-Water Caribbean Sponge of the Genus Batzella. J. Nat. Prod. 1999, 62, 1208-1211.

24. Copp, B.R.; Ireland, C.M. Wakayin: A Novel Cytotoxic Pyrroloiminoquinone Alkaloid from the Ascidian Clavelina Species. J. Org. Chem. 1991, 56, 4596-4597.

25. Stierle, D.B.; Faulkner, D.J. Two New Pyrroloquinoline Alkaloids from the Sponge Damiria sp. $J$. Nat. Prod. 1991, 54, 1131-1133.

26. Schmidt, E.W.; Harper, M.K.; Faulkner, D.J. Makaluvamines H-M and Damirone C from the Pohnpeian Sponge Zyzzya Fuliginosa. J. Nat. Prod. 1995, 58, 1861-1867.

27. Delfourne, E. Analogues of Marine Pyrroloiminoquinone Alkaloids: Synthesis and Antitumore Properties. Anti-Cancer Agents Med. Chem. 2008, 8, 910-916.

28. Kobayashi, J.; Cheng, J.F.; Ishibashi, M.; Nakamura, H.; Ohizumi, Y.; Hirata, Y.; Sasaki, T.; Lu, H.; Clardy, J. Prianosin A, a novel antileukemic alkaloid from the okinawan marine sponge Prianos melanos. Tetrahedron Lett. 1987, 28, 4939-4942.

29. Perry, N.B.; Blunt, J.W.; Munro, M.H.G. Cytotoxic pigments from new zealand sponges of the genus latrunculia : discorhabdins A, B and C. Tetrahedron 1988, 44, 1727-1734.

30. Perry, N.B.; Blunt, J.W.; Munro, M.H.G. Discorhabdin D, an antitumor alkaloid from the sponges Latrunculia brevis and Prianos sp. J. Org. Chem. 1988, 53, 4127-4128.

31. Dijoux, M.G.; Gamble, W.R.; Hallock, Y.F.; Cardellina II, J. H.; Soest, R.; Boyd, M. R. A New Discorhabdin from Two Sponge Genera. J. Nat. Prod. 1999, 62, 636-637.

32. Ford, J.; Capon, R.J. Discorhabdin R: A New Antibacterial Pyrroloiminoquinone from Two Latrunculiid Marine Sponges, Latrunculia sp. and Negombata sp. J. Nat. Prod. 2000, 63, 1527-1528.

33. El-Naggar, M.; Capon, R.J. Discorhabdins Revisited: Cytotoxic Alkaloids from Southern Australian Marine Sponges of the Genera Higginsia and Spongosorites. J. Nat. Prod. 2009, 72, 460-464. 
34. Cheng, J.; Ohizumi, Y.; Wälchli, M.R.; Nakamura, H.; Hirata, Y.; Sasaki, T.; Kobayashi, J. Prianosins B, C, and D, novel sulfur-containing alkaloids with potent antineoplastic activity from the Okinawan marine sponge Prianos melanos. J. Org. Chem. 1988, 53, 4621-4624.

35. Lang, G.; Pinkert, A.; Blunt, J.W.; Munro, M.H.G. Discorhabdin W, the First Dimeric Discorhabdin. J. Nat. Prod. 2005, 68, 1796-1798.

36. Blunt, J.W.; Munro, M.H.G.; Battershill, C.N.; Copp, B.R.; McCombs, J.D.; Perry, N.B.; Prinsep, M.; Thompson, A.M. From the Antarctic to the Antipodes; $45^{\circ}$ of marine chemistry. New J. Chem. 1990, 14, 761-775.

37. Kita, Y.; Tohma, H.; Inagaki, M.; Hatanaka, K.; Yakura, T. Total synthesis of discorhabdin C: a general aza spiro dienone formation from $O$-silylated phenol derivatives using a hypervalent iodine reagent. J. Am. Chem. Soc. 1992, 114, 2175-2180.

38. Kita, Y.; Egi, M.; Tohma, H. Total synthesis of sulfur-containing pyrroloiminoquinone marine product, ( \pm )-makaluvamine $\mathrm{F}$ using hypervalent iodine(III)-induced reactions. Chem. Commun. 1999, 143-144.

39. Kita, Y.; Egi, M.; Takada, T.; Tohma, H. Development of novel reactions using hypervalent iodine(III) reagents. Total synthesis of sulfur-containing pyrroloiminoquinone marine product, (士)-makaluvamine F. Synthesis 1999, 885-897.

40. Tohma, H.; Harayama, Y.; Hashizume, M.; Iwata, M.; Egi, M.; Kita, Y. Synthetic studies on the sulfur-cross-linked core of antitumor marine alkaloid, discorhabdins: total synthesis of discorhabdin A. Angew. Chem. Int. Ed. 2002, 41, 348-350.

41. Tohma, H.; Harayama, Y.; Hashizume, M.; Iwata, M.; Kiyono, Y.; Egi, M.; Kita, Y. The First Total Synthesis of Discorhabdin A. J. Am. Chem. Soc. 2003, 125, 11235-11240.

42. Harayama, Y.; Yoshida, M.; Kamimura, D.; Kita, Y. The novel and efficient direct synthesis of N,O-acetal compounds using a hypervalent iodine(III) reagent: an improved synthetic method for a key intermediate of discorhabdins. Chem. Commun. 2005, 1764-1766.

43. Harayama, Y.; Yoshida, M.; Kamimura, D.; Wada, Y.; Kita, Y. The efficient direct synthesis of $\mathrm{N}, \mathrm{O}$-acetal compounds as key intermediates of discorhabdin A: oxidative fragmentation reaction of $\alpha$-amino acids or $\beta$-amino alcohols by using hypervalent iodine(III) reagents. Chem. Eur. J. 2006, 12, 4893-4899.

44. Wada, Y.; Otani, K.; Endo, N.; Harayama, Y.; Kamimura, D.; Yoshida, M.; Fujioka, H.; Kita, Y. The first total synthesis of prianosin B. Tetrahedron 2009, 65, 1059-1062.

45. Perry, N.B.; Blunt, J.W.; McCombs, J.D.; Munro, M.H.G. Discorhabdin C, a highly cytotoxic pigment from a sponge of the genus Latrunculia. J. Org. Chem. 1986, 51, 5476-5478.

46. Kita, Y.; Yakura, T.; Tohma, H.; Kikuchi, K.; Tamura, Y. A synthetic approach to discorhabdin alkaloids: Hypervalent iodine oxidation of $p$-substituted phenol derivatives to azacarbocyclic spirodienones. Tetrahedron Lett. 1989, 30, 1119-1120.

47. Nishiyama, S.; Cheng, J.F.; Tao, X.L.; Yamamura, S. Synthetic studies on novel sulfur-containing alkaloids, prianosins and discorhabdins: total synthesis of discorhabdin C. Tetrahedron Lett. 1991, 32, 4151-4154.

48. Tao, X.L.; Cheng, J.F.; Nishiyama, S.; Yamamura, S. Synthetic studies on tetrahydropyrroloquinoline-containing natural products: Syntheses of discorhabdin C, batzelline C and isobatzelline C. Tetahedron 1994, 50, 2017-2028. 
49. Aubart, K.M.; Heathcock, C.H. A Biomimetic Approach to the Discorhabdin Alkaloids: Total Syntheses of Discorhabdins C and E and Dethiadiscorhabdin D. J. Org. Chem. 1999, 64, 16-22.

50. Kita, Y.; Egi, M.; Ohtsubo, M.; Saiki, T.; Okajima, A.; Takada, T.; Tohma, H. Hypervalent iodine(III)-induced intramolecular cyclization reaction of substituted phenol ethers with an alkyl azido side-chain: a novel and efficient synthesis of quinone imine derivatives. Chem. Pharm. Bull. 1999, 47, 241-245.

51. Kita, Y.; Egi, M.; Okajima, A.; Ohtsubo, M.; Takada, T.; Tohma, H. Hypervalent iodine(III) induced intramolecular cyclization of substituted phenol ethers bearing an alkyl azido sidechain-a novel synthesis of quinone imine ketals. Chem. Commun. 1996, 1491-1492.

52. Kita, Y.; Egi, M.; Ohtsubo, M.; Saiki, T.; Takada, T.; Tohma, H. Novel and efficient synthesis of sulfur-containing heterocycles using a hypervalent iodine(III) reagent. Chem. Commun. 1996, 2225-2226.

53. Tohma, H.; Egi, M.; Ohtsubo, M.; Watanabe, H.; Takizawa, S.; Kita, Y. A novel and direct $\alpha-$ azidation of cyclic sulfides using a hypervalent iodine(III) reagent. Chem. Commun. 1998, 173-174.

54. Kita, Y.; Takada, T.; Mihara, S.; Tohma, H. A novel and direct sulfenylation of phenol ethers using phenyliodine(III) bis(trifluoroacetate) (PIFA) and various thiophenols. Synlett 1995, 211-212.

55. Kita, Y.; Takada, T.; Mihara, S.; Whelan, B.A.; Tohma, H. Novel and Direct Nucleophilic Sulfenylation and Thiocyanation of Phenol Ethers Using a Hypervalent Iodine(III) Reagent. $J$. Org. Chem. 1995, 60, 7144-7148.

56. Lill, R.E.; Major, D.A.; Blunt, J.W.; Munro, M.H.G. Studies on the Biosynthesis of Discorhabdin B in the New Zealand Sponge Latrunculia sp. B. J. Nat. Prod. 1995, 58, 306-311.

57. Grkovic, T.; Kaur, B.; Webb, V.L.; Copp, B.R. Semi-synthetic preparation of the rare, cytotoxic, deep-sea sourced sponge metabolites discorhabdins $\mathrm{P}$ and U. Bioorg. Med. Chem. Lett. 2006, 16, 1944-1946.

58. Miller, K.; Alvarez, B.; Bettershill, C.; Northcote, P.; Parthasarathy, H. Genetic, morphological, and chemical divergence in the sponge genus Latrunculia (Porifera: Demospongiae) from New Zealand. Mar. Biol. 2001, 139, 235-250.

59. Izawa, T.; Nishiyama, S.; Yamamura, S. Total syntheses of makaluvamines A, B, C, D and E, cytotoxic pyrroloiminoquinone alkaloids isolated from marine sponge bearing inhibitory activities against topoisomerase II. Tetrahedron 1994, 50, 13593-13600.

60. White, J.D.; Yager, K.M.; Yakura, T. Synthetic Studies of the Pyrroloquinoline Nucleus of the Makaluvamine Alkaloids. Synthesis of the Topoisomerase II Inhibitor Makaluvamine D. J. Am. Chem. Soc. 1994, 116, 1831-1838.

61. Patel, S.P.; Nadkarni, D.H.; Murugesan, S.; King, J.R.; Velu, S.E. Azide-Mediated Detosylation of $N$-Tosylpyrroloiminoquinones and $N$-Tosylindole-4,7-quinones. Synlett 2008, 2864-2868.

62. Regitz, M. Recent synthetic methods in diazo chemistry. Synthesis 1972, 351-373.

63. Evans, D.A.; Britton, T.C. Electrophilic azide transfer to chiral enolates. A general approach to the asymmetric synthesis of .alpha.-amino acids. J. Am. Chem. Soc. 1987, 109, 6881-6883.

64. Weil, T.; Cais, M.A Simplified Procedure for the Preparation of Diazocyclopentadiene and Some Related Compounds. J. Org. Chem. 1963, 28, 2472. 
65. Regitz, M.; Liedhegener, A. Reaktionen aktiver methylenverbindungen mit aziden-XV $\stackrel{* 1}{\text { : }}$ Synthese von diazocyclopentadienen durch diazogruppenübertragung und einige reaktionen. Tetrahedron 1967, 23, 2701-2708.

66. Wada, Y.; Otani, K.; Endo, N.; Harayama, Y.; Kamimura, D.; Yoshida, M.; Fujioka, H.; Kita, Y. Synthesis of Antitumor Marine Alkaloid Discorhabdin A Oxa Analogues. Org. Lett. 2009, 11, 4048-4050.

67. Radisky, D.C.; Radisky, E.S.; Barrows, L.R.; Copp, B.R.; Kramer, R.A.; Ireland, C.M. Novel cytotoxic topoisomerase II inhibiting pyrroloiminoquinones from Fijian sponges of the genus Zyzzya. J. Am. Chem. Soc. 1993, 115, 1632-1638.

68. Dijoux, M.-G.; Schnabel, P.C.; Hallock, Y.F.; Boswell, J.L.; Johnson, T.R.; Wilson, J.A.; Ireland, C.M.; Soest, R.; Boyd, M.R.; Barrows, L.R.; Cardellina, J.H. Antitumor activity and distribution of pyrroloiminoquinones in the sponge genus Zyzzya. Bioorg. Med. Chem. 2005, 13, 6035-6044.

69. Shinkre, B.A.; Raisch, K.P.; Fan, L.; Velu, S.E. Analogs of the marine alkaloid makaluvamines: Synthesis, topoisomerase II inhibition, and anticancer activity. Bioorg. Med. Chem. Lett. 2007, 17, 2890-2893.

70. Passarella, D.; Belinghieri, F.; Scarpellini, M.; Pratesi, G.; Zunino, F.; Gia, O.M.; Via, L.D.; Santoro, G.; Danieli, B. Analogs of the marine alkaloid makaluvamines: Synthesis, topoisomerase II inhibition, and anticancer activity. Bioorg. Med. Chem. 2008, 16, 2431-2438.

71. Shinkre, B.A.; Raisch, K.P.; Fan, L.; Velu, S.E. Synthesis and antiproliferative activity of benzyl and phenethyl analogs of makaluvamines. Bioorg. Med. Chem. 2008, 16, 2541-2549.

Samples Availability: Available from the authors.

(C) 2010 by the authors; licensee MDPI, Basel, Switzerland. This article is an open-access article distributed under the terms and conditions of the Creative Commons Attribution license (http://creativecommons.org/licenses/by/3.0/). 